\title{
How synaptic strength, short-term plasticity, and temporal factors contribute to neuronal spike output
}

\author{
Alexandra Gastone Guilabert ${ }^{1}$, Benjamin Ehret ${ }^{1}$, Moritz O. Buchholz ${ }^{1}$, Gregor F.P. Schuhknecht ${ }^{1,2} \bowtie$
}

\author{
1 Institute of Neuroinformatics, University of Zurich and ETH Zurich, Zurich, Switzerland \\ 2 Department of Molecular and Cellular Biology, Harvard University, Cambridge, MA, USA \\ $\bowtie$ Correspondence should be addressed to gregor_schuhknecht@fas.harvard.edu
}

\begin{abstract}
To compute spiking responses, neurons integrate inputs from thousands of synapses whose strengths span an order of magnitude. Intriguingly, in mouse neocortex, the small minority of 'strong' synapses is found predominantly between similarly tuned cells, suggesting they are the synapses that determine a neuron's spike output. This raises the question of how other computational primitives, such as 'background' activity from the majority of synapses, which are 'weak', short-term plasticity, and temporal synchrony contribute to spiking. First, we combined extracellular stimulation and whole-cell recordings in mouse barrel cortex to map the distribution of excitatory postsynaptic potential (EPSP) amplitudes and paired-pulse ratios of excitatory synaptic connections converging onto individual layer 2/3 (L2/3) neurons. While generally net short-term plasticity was weak, connections with EPSPs $>2 \mathrm{mV}$ displayed pronounced paired-pulse depression. EPSP amplitudes and paired-pulse ratios of connections converging onto the same neurons spanned the full range observed across $L 2 / 3$ and there was no indication that strong synapses nor those with particular short-term plasticity properties were associated with particular cells, which critically constrains theoretical models of cortical filtering. To investigate how different computational primitives of synaptic information processing interact to shape spiking, we developed a computational model of a pyramidal neuron in the rodent $L 2 / 3$ circuitry: firing rates and pairwise correlations of presynaptic inputs were constrained by in vivo observations, while synaptic strength and short-term plasticity were set based on our experimental data. Importantly, we found that the ability of strong inputs to evoke spiking critically depended on their high temporal synchrony and high firing rates observed in vivo and on synaptic background activity - and not primarily on synaptic strength, which in turn further enhanced information transfer. Depression of strong synapses was critical for maintaining a neuron's responsivity and prevented runaway excitation. Our results provide a holistic framework of how cortical neurons exploit complex synergies between temporal coding, synaptic properties, and noise in order to transform synaptic inputs into output firing.
\end{abstract}




\section{Introduction}

Pyramidal neurons in neocortex compute spiking responses on the basis of synaptic inputs they receive asynchronously from thousands of neurons in the surrounding brain tissue. The strengths of these inputs span one order of magnitude and typically follow a lognormal distribution: while the majority of synaptic connections evoke small excitatory postsynaptic potentials (EPSPs), a small minority elicits comparably large EPSPs (Markram et al., 1997; Tarczy-Hornoch et al., 1999; Song et al., 2005; Feldmeyer et al., 2006; Buzsáki and Mizuseki, 2014; Cossell et al., 2015). Intriguingly, in mouse primary visual cortex (V1), such 'strong' connections were found to occur predominantly between those neurons that also exhibit the most similar receptive field properties in vivo (Cossell et al., 2015). From these observations, a simple organizational principle of synaptic strength was proposed, in which the majority of the synaptic excitation necessary for action potential firing is provided by a small fraction of strong synaptic inputs, which determine the spike output of the postsynaptic neuron (Cossell et al., 2015). The notion that synaptic strength is the primary determinant for the functional properties of neocortical circuits is attractive because it suggests that mapping the strongest connections in functional or structural analyses reveals the true underlying functional organization of neocortical circuits. However, a more complex picture recently emerged from ferret $\mathrm{V} 1$, where the response selectivity of neurons to visual stimulation was found to be determined by the cumulative weight of all co-active synapses, and could not simply be predicted from the tuning of synapses with large EPSPs (Scholl et al., 2020).

Several other observations give further weight to the notion that synaptic strength alone is insufficient to explain neuronal response properties. Synapses are complex biophysical devices, whose response during ongoing activation is insufficiently captured by only a single weight parameter. It is intriguing that those cortical synapses that elicit the largest EPSPs also tend to exhibit the most pronounced short-term depression (Reyes and Sakmann, 1999; Jouhanneau et al., 2015; Lefort and Petersen, 2017), which can vastly reduce the total charge a synapse can deliver to its postsynaptic partner during repeated activation (Stratford et al., 1996; Castro-Alamancos and Oldford, 2002; Chung et al., 2002; Abbott and Regehr, 2004; Boudreau and Ferster, 2005; Bruno and Sakmann, 2006). Thus, synaptic connections with large EPSPs recorded in vitro operate in a significantly depressed state in vivo due to ongoing spontaneous and stimulus-evoked activation (Boudreau and Ferster, 2005). Furthermore, even the largest EPSP amplitudes provide only a small fraction of the depolarizing charge necessary to drive the membrane potential of a cortical neuron through the spike threshold. Thus, temporal coincidence in presynaptic spike trains must necessarily be an important factor for information coding in neocortex (Bruno and Sakmann, 2006; Banitt et al., 2007; Wang et al., 2010; Schoonover et al., 2014; Scholl et al., 2020). Finally, neurons in vivo operate in the presence of significant synaptic background activity. Spontaneous firing rates of pyramidal cells in the superficial layers of rodent sensory areas range between 0.08 to $0.39 \mathrm{~Hz}$ in vivo (Waters and Helmchen, 2006; de Kock et al., 2007; Kerr et al., 2007; de Kock and Sakmann, 2009; Niell and Stryker, 2010, 2010; O'Connor et al., 2010). Because pyramidal neurons in rodent sensory areas are estimated to receive input from up to 8000 synapses (Schüz and Palm, 1989), they must experience hundreds to thousands of spontaneous synaptic events per second. In rodent V1, synaptic connections with small EPSPs occur predominantly between cells that display different response properties and thus fire with little temporal synchrony 
during visual stimulation (Cossell et al., 2015). Thus, in rodent sensory areas, the vast majority of excitatory synapses formed with any given pyramidal neuron provide a constant bombardment of excitation that seems relatively unrelated to the tuning of that neuron. Therefore, in order to compute spiking responses from their synaptic inputs, neocortical neurons operate in a complex parameter space. While much research has been conducted on the computational role of synaptic strength [e.g. (Lefort et al., 2009; Cossell et al., 2015; Scholl et al., 2020)], short-term plasticity [e.g. (Abbott et al., 1997; Castro-Alamancos and Oldford, 2002; Chung et al., 2002; Banitt et al., 2007; Rothman et al., 2009; Díaz-Quesada et al., 2014)], and the temporal structure within synaptic inputs [e.g. (Bruno and Sakmann, 2006; Banitt et al., 2007; Wang et al., 2010; Schoonover et al., 2014)], it remains much less studied how these parameters act together to shape information transfer in sensory areas.

Here, we combined experimental work and computational modeling to investigate systematically how this complex parameter-space could shape the spiking responses of pyramidal neurons in L2/3 of mouse barrel cortex (S1). The distributions and patterns of action potential firing rates (de Kock et al., 2007; de Kock and Sakmann, 2009; Sakata and Harris, 2009; O'Connor et al., 2010), synaptic strength (Lefort et al., 2009; Cossell et al., 2015; Seeman et al., 2018), correlations within neuronal activity (Kerr et al., 2007; Sato et al., 2007), and temporal correlations within synaptic inputs converging onto the same neuron (Cossell et al., 2015) have been well-characterized for L2/3 in rodent sensory areas in vivo. However, even though paired-pulse ratios have been measured for excitatory synapses across all cortical layers and different areas and species, most studies relied on small datasets that aimed to detect general differences in the mean (Reyes and Sakmann, 1999; Feldmeyer et al., 2006; Costa et al., 2013; Jouhanneau et al., 2015; Lefort and Petersen, 2017; Seeman et al., 2018). Thus, a detailed characterization of the exact statistical distribution of short-term plasticity in mouse sensory $L 2 / 3$ is missing. Likewise, the relationship between synaptic strength and short-term plasticity has not been characterized clearly for L2/3. Finally, it remains unknown whether synaptic connections that converge onto the same neuron exhibit a systematic bias of EPSP amplitudes (Koulakov et al., 2009) or short-term plasticity, which could endow individual neurons with low-pass filter or high-pass filter properties, if they were to receive predominantly depressing or facilitating synapses, respectively (Chance et al., 1998; Fortune and Rose, 2000, 2001; Abbott and Regehr, 2004). We addressed these questions by combining whole-cell recordings of $L 2 / 3$ pyramidal neurons in barrel cortex slices with extracellular stimulation of axons of passage. Then, we developed a computational model of a L2/3 pyramidal neuron that received excitatory inputs from 270 other L2/3 neurons (Sarid et al., 2013), whose synaptic strengths and short-term plasticity were modeled after our experimental data. Presynaptic inputs were set to display temporal firing patterns constrained by in vivo data: the few synaptic connections eliciting large EPSPs fired temporally correlated spikes at high frequencies and were termed 'strong' inputs, while the more numerous connections triggering small EPSPs - termed 'weak' inputs - fired uncorrelated spikes at lower frequencies (Cossell et al., 2015). By selectively manipulating the relationship between synaptic strength, short-term plasticity, and temporal structure in the synaptic inputs, we characterized the importance of each of these parameters and their interdependencies in our simulation. 


\section{Results}

\section{Mapping synaptic strength and short-term plasticity in L2/3}

We characterized the distribution of EPSP amplitudes and corresponding paired-pulse ratios of excitatory synaptic connections formed with neurons in barrel cortex L2/3. To do so, we measured somatic whole-cell responses of $L 2 / 3$ neurons to extracellular paired-pulse stimulation at multiple locations in the surrounding L2/3 (Fig. 1 A, B). We obtained recordings from 20 regular-spiking neurons (i.e., pyramidal neurons, as confirmed by post-hoc histology, see Methods) for which we identified a total of 74 sites at which extracellular stimulation evoked EPSPs (mean of 3.7 synaptic connections per neuron) and of 4 fast-spiking neurons (i.e., interneurons, as confirmed by post-hoc histology) for which we found a total of 17 extracellular stimulation sites (4.3 synaptic connections per neuron). Thus, our complete dataset contained 91 evoked EPSPs recorded across 24 L2/3 neurons.

The distribution of peak amplitudes across the 74 EPSPs recorded in regular-spiking cells ranged from $0.29 \mathrm{mV}$ to $4.15 \mathrm{mV}$ (mean \pm s.d.: $1.23 \pm 0.75 \mathrm{mV}$ ), was markedly right-skewed, and could be fit well with a lognormal distribution $\left(R^{2}=0.97\right)$ (Fig. $\left.1 \mathrm{D}\right)$. The mean coefficient of variation was 0.19 \pm 0.06 , the mean EPSP onset latency was $2.14 \pm 1.12$ ms and the mean $10-90 \%$ rise time was 2.54 $\pm 0.86 \mathrm{~ms}$. For all 74 synaptic connections, we also recorded the paired-pulse ratio at an inter-spike interval of $20 \mathrm{~ms}$. Interestingly, the distribution of paired-pulse ratios appeared noticeably symmetrical with a mean \pm s.d. of $0.93 \pm 0.20$ and could be fit well with a normal distribution $\left(R^{2}=0.93\right)$ (Fig. $\left.1 \mathrm{E}\right)$.

A similar picture emerged for the 17 EPSPs recorded in the fast-spiking cells: their amplitudes ranged from $0.52 \mathrm{mV}$ to $3.03 \mathrm{mV}$ (mean \pm s.d.: $1.49 \pm 0.75 \mathrm{mV}$ ) and were best captured by a lognormal distribution $\left(R^{2}=0.65\right)($ Fig. $1 \mathrm{D})$. The mean coefficient of variation was $0.18 \pm 0.06$, the mean onset latency was $2.44 \pm 0.99 \mathrm{~ms}$, and the $10-90 \%$ rise time was $0.83 \pm 0.4 \mathrm{~ms}$. The distribution of corresponding paired-pulse ratios was also markedly symmetrical with a mean of $0.87 \pm 0.25$ (Fig. $1 \mathrm{E}$ ) and could be fit well with a normal distribution $\left(R^{2}=0.69\right)$.

Given their different symmetries (lognormal versus Gaussian, respectively), the question arose of how EPSP amplitudes and their corresponding paired-pulse ratios could be mapped onto one another, i.e., whether there was a systematic relationship between synaptic strength and short-term plasticity. Interestingly, a scatter plot of the response amplitudes to the $2^{\text {nd }}$ stimulation pulse against the response amplitudes to the $1^{\text {st }}$ stimulation pulse (corresponding to the EPSP amplitude) showed the tendency that synaptic connections with larger EPSPs were depressing, while connections with smaller EPSPs exhibited a range of facilitating and depressing paired-pulse ratios (Fig. $1 \mathrm{C}, \mathrm{F}$ ). There was no significant correlation in our dataset between EPSP amplitude and short-term plasticity for connections formed with either regular-spiking or fast-spiking neurons. A significant negative correlation only emerged when we pooled all synaptic connections recorded in the study $(r=-0.36, p=0.0004, n=91$, non-parametric Spearman correlation coefficient). Thus, EPSP amplitude and short-term plasticity appeared to be only very weakly correlated across a large number of synaptic connections in L2/3. To investigate this further, we binned our dataset of synaptic connections recorded in regular-spiking neurons depending on their EPSP amplitude (into $0.5 \mathrm{mV}$ bins, not shown). Critically, we found that in all bins with EPSP amplitudes below $2 \mathrm{mV}$, synaptic connection displayed a range of facilitating and depressing paired- 
bioRxiv preprint doi: https://doi.org/10.1101/2021.12.21.473708; this version posted December 23, 2021. The copyright holder for this preprint (which was not certified by peer review) is the author/funder, who has granted bioRxiv a license to display the preprint in perpetuity. It is made available under aCC-BY-NC-ND 4.0 International license.

pulse ratios. By contrast, all connections with EPSP amplitudes above $2 \mathrm{mV}$ were depressing $(\mathrm{n}=10)$ (Fig. $1 \mathrm{~F}$ ). This indicated that an EPSP amplitude of $2 \mathrm{mV}$ constituted a natural divider of synaptic strength in our dataset. When we split the dataset accordingly, we found that connections below $2 \mathrm{mV}$ had a mean paired-pulse ratio of $0.95 \pm 0.20$ (i.e., exhibiting little net short-term plasticity), while connections above $2 \mathrm{mV}$ had a significantly lower mean paired-pulse ratio of $0.83 \pm 0.10$ (Fig. $1 \mathrm{~F}$ ).

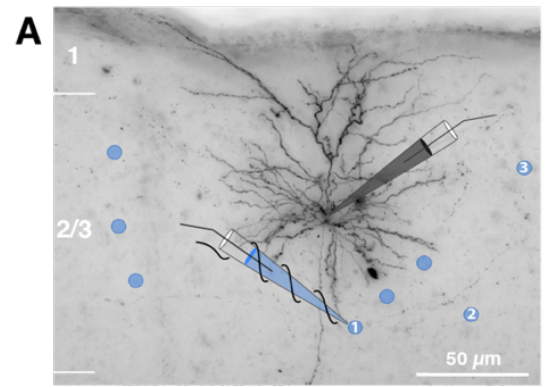

B

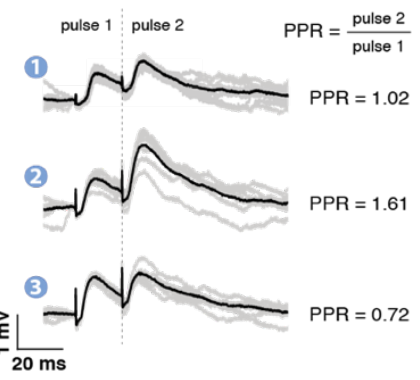

C

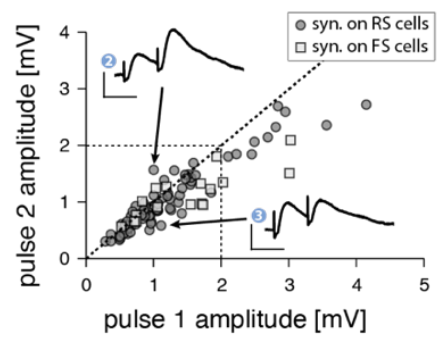

D

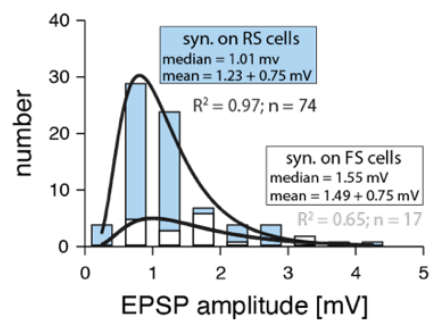

E

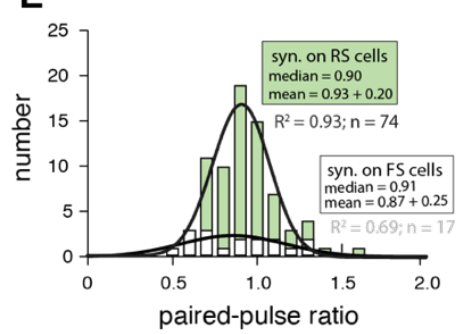

$\mathbf{F}$

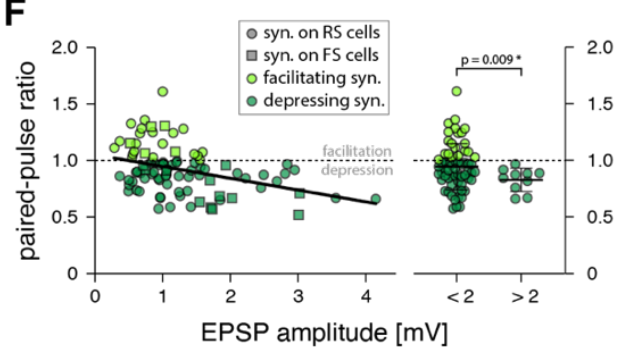

Figure 1. EPSP amplitudes and paired-pulse ratios of excitatory synaptic connections in barrel cortex L2/3.

A Example of recorded regular-spiking L2/3 neuron in mouse barrel cortex visualized through post-hoc biocytin histology. Blue dots indicate locations of successful extracellular stimulation, blue pipette signifies extracellular stimulation electrode. The neuron's responses to stimulation at three different positions (labeled 1-3) are shown in B.

B Somatic voltage recordings following $20 \mathrm{~ms}$ paired-pulse stimulation in the locations indicated by numbers. Grey traces, individual trials; black traces, average response; paired-pulse ratios (PPR) indicated. For timing of extracellular stimulation pulses, note the electrical stimulation artifact in somatic voltage responses.

C Scatter plot showing, for all recorded excitatory synaptic connections, the responses to the second pulse versus the response to the first pulse (corresponding to the EPSP amplitude) of the paired-pulse stimulation paradigm. Circles, synaptic connections formed with regular-spiking (RS) neurons; squares, connections formed with fast-spiking neurons (FS). Data points below diagonal indicate depressing synaptic connections, dots above diagonal indicate facilitating connections. Voltage traces, same as traces 2 and 3 in B with identical same scale bars.

D Distribution of EPSP amplitudes recorded in regular-spiking (blue) and fast-spiking L2/3 (white) L2/3 neurons; histograms were fit with lognormal functions ( $R^{2}$, goodness of fit).

E Distribution of paired-pulse ratios recorded in regular-spiking (green) and fast-spiking L2/3 (white) L2/3 neurons; histograms were fit with Gaussian functions ( $R^{2}$, goodness of fit).

F Left, scatter plot showing relationship of EPSP amplitude and paired-pulse ratio for excitatory synaptic connections formed with regular-spiking (circles, $n=74$ ) and fast-spiking (squares, $n=17$ ) cells; light green, facilitating connections; dark green, depressing connections. Line was fit to all datapoints with linear regression (slope: $=-0.104, R^{2}=0.144$ ). Right, comparison of paired-pulse ratios of synaptic connections formed with regular-spiking neurons that were binned into 'small' (EPSP $<2 \mathrm{mV} ; n=$ 64 ) and 'large' (EPSP > $2 \mathrm{mV} ; \mathrm{n}=10$ ) synaptic connections (parametric Welch's $\mathrm{t}$ test). 


\section{No clustering of connections with similar paired-pulse ratios on $L 2 / 3$ neurons}

Next, we investigated whether EPSP amplitudes and short-term plasticity across those synaptic connections formed with the same regular-spiking L2/3 neurons followed the same distributions as those of all 74 connections across all regular-spiking neurons. Alternatively, the synaptic inputs onto a given cortical neuron may be statistically correlated, i.e. individual neurons could receive synaptic connections with systematically biased EPSP amplitudes or paired-pulse ratios that deviate from the overall distributions found across $L 2 / 3$, which may constitute a mechanism to endow individual cells with high-pass or low-pass filtering properties (Fortune and Rose, 2001; Abbott and Regehr, 2004). For a total of 8 regular-spiking neurons, we were able to characterize at least 5 different afferent synaptic connections (47 connections in total, mean of 5.9 connections per cell). We will refer to the distribution of paired-pulse ratios and EPSP amplitudes across all our recorded synapses as the "population distribution" and to the distributions of paired-pulse ratios and EPSPs of synaptic connections converging onto a single cell as "cell distributions". We used the non-parametric Kolmogorov-Smirnov test to detect if there was a significant difference between the respective cell distributions and the population distribution. Interestingly, for all 8 cells, the cell distributions were not significantly different from the population distribution for both EPSP amplitude and paired-pulse ratios (Fig. 2 A, B).

Precise quantification of synaptic short-term plasticity requires electrophysiological recordings. Using whole-cell patch-clamp recordings in combination with minimal stimulation of axons of passage, however, limits the number of synaptic connections that can be recorded for any given neuron, yielding low statistical power on the level of individual cells. Therefore, we conducted a power analysis to estimate the detectable effect sizes in our dataset (see Methods for details). For detecting a significant $(\alpha=0.05)$ difference between each of the 8 paired-pulse ratio cell distributions and the population distribution, the Kolmogorov-Smirnov test had an average power of $17 \%$ for an effect size of 0.1 , a power of $53 \%$ for an effect size of 0.2 , and a power of $85 \%$ for an effect size of 0.3 , where effect size corresponds to a systematic difference in the means of the cell distributions. Thus, the statistical power was low on the level of individual experiments. Because we could repeat the experiment 8 times, however, even small systematic differences between cell distributions and population distribution, while undetectable in single experiments, should have been revealed in at least one or a few of the 8 neurons we recorded from. To investigate this further, we used a binomial model (see Methods) to assess the power of the entire experimental series by asking: what systematic difference in paired-pulse ratios should have been observed in at least one of the 8 experiments at the $95 \%$ significance level? We found that the probability to detect a significant difference across our entire dataset was $78 \%$ for an effect size of 0.1 and $99.7 \%$ for an effect size of 0.2 , with the $95 \%$ significance level at an effect size of 0.15 . Critically, an effect size of 0.15 is below the paired-pulse ratio difference of 0.16 that we detected between the small- and large-EPSP connections formed with pyramidal neurons in L2/3 (Fig. $2 \mathrm{C}$ ). Thus, our experimental series achieved the statistical power necessary to detect differences in paired-pulse ratios at physiological magnitudes that we found to exist in L2/3. This suggests that shortterm plasticity of excitatory synapses formed with individual regular-spiking cortical neurons in L2/3 spans the full range observed in $L 2 / 3$ and is not markedly functionally clustered on the level of single neurons. 
Likewise, for detecting a significant difference between each of the 8 EPSP cell distributions and the population distribution, the Kolmogorov-Smirnov test had an average power of $4.9 \%$ for an effect size of $0.2 \mathrm{mV}$, a power of $15 \%$ for an effect size of $0.4 \mathrm{mV}$, and a power of $46 \%$ for an effect size of $0.6 \mathrm{mV}$. Analogous Monte Carlos simulations showed that the probability of detecting a significant difference in the mean EPSP amplitudes across our entire dataset was $72 \%$ for a systematic effect size of $0.4 \mathrm{mV}$ and $99.3 \%$ for a systematic effect size of $0.6 \mathrm{mV}$, with the $95 \%$ significance level at $0.52 \mathrm{mV}$ (Fig. $2 \mathrm{C}$ ). In summary, these are important experimental results that contradict the theory-inspired hypothesis that synaptic inputs onto single cortical neurons may be statistically correlated (Koulakov et al., 2009).
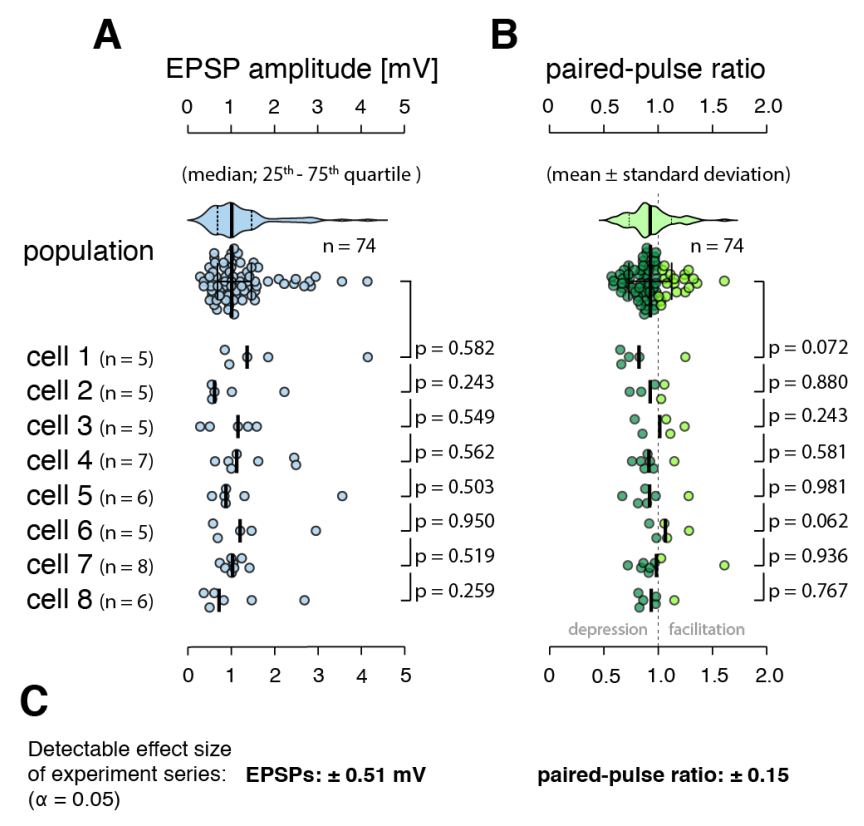

Figure 2. Excitatory synaptic connections formed with regular-spiking L2/3 neurons do not exhibit a systematic clustering of EPSP amplitude and short-term plasticity.

A Top, distribution of EPSP amplitudes recorded across all regular-spiking neurons (population distribution). Bottom, distributions of the EPSP amplitudes across the 8 regular spiking neurons, for which at least 5 synapses were found (cell distributions). N, number of synapses recorded per cell; $p$, non-parametric Kolmogorov-Smirnov test between each cell distribution and the population distribution, medians are indicated.

B Representation of short-term plasticity data, panel layout as in A; light green, facilitating synaptic connections; dark green, depressing connections, means are indicated.

C Estimation of the effect sizes that are detectible across the experimental series at a $5 \%$ significance level.

\section{Modeling the interplay of synaptic strength, short-term plasticity, and temporal input structure}

We generated a conductance-based model of a L2/3 pyramidal neuron to investigate how synaptic strength, short-term plasticity, and temporal structure in synaptic inputs interact within the L2/3 circuitry to shape the response properties of cortical neurons (Fig. $3 A-C$ ). The model neuron received excitatory inputs from 270 presynaptic neurons (Feldmeyer et al., 2006; Sarid et al., 2013), whose synaptic weights (Fig. $3 \mathrm{D}$ ) and short-term plasticity properties (Fig. $3 \mathrm{E}$ ) were constrained following our 
extracellular stimulation experiments (see Methods). Note that this number of presynaptic L2/3 cells is based on the assumption that L2/3 neurons form on average 3 anatomical synapses with their postsynaptic partners in L2/3 (Feldmeyer et al., 2006; Sarid et al., 2013). In our model, this is captured by the fact that the axons of passage we activated with minimal stimulation must have also formed multiple synapses with the recorded neurons on average. This is evident when comparing the range of EPSP amplitudes we recorded with minimal stimulation $(0.29-4.15 \mathrm{mV})$ with EPSP amplitudes obtained from paired recordings $(0.15-2.25 \mathrm{mV})$, for which the number of anatomical synapses per connection (mean of 1.6) was additionally established from EM (Holler et al., 2021).

The temporal input correlations (Cossell et al., 2015) (Fig. $3 \mathrm{~F}$ ) and the firing rates (O'Connor et al., 2010) (Fig. $3 \mathrm{G}$ ) across the 270 synaptic inputs were constrained by published in vivo data for rodent cortex (see Methods), such that a small number of strong synaptic inputs fired temporally correlated spikes at high frequencies and exhibited large EPSP amplitudes and corresponding shortterm depression. The remaining majority of weak synapses, providing 'background' activity, were set to fire at low frequencies and in a temporally uncorrelated pattern, resembling a random Poisson process, and exhibited low EPSP amplitudes without pronounced net short-term plasticity (Fig. 3 A, B).

After the model was set up in this manner, we verified that all parameters were distributed following experimental data and that the interdependencies between EPSP amplitude and short-term plasticity and EPSP amplitude and temporal correlation structure (Cossell et al., 2015) were preserved (Fig. 4). The model EPSP amplitude distribution (Fig. 3 D, Fig. 4 A; mean \pm s.d.: $1.23 \pm 0.69 \mathrm{mV}, \mathrm{n}=270$ ) and paired-pulse ratio distribution for a $20 \mathrm{~ms}$ paired-pulse interval (Fig. 3 E, Fig. 4 B; mean \pm s.d.: $0.91 \pm$ $0.19, n=270$ ) did not differ from the distributions we had measured in regular-spiking neurons in vitro ( $p=0.90$ and $p=0.67$, respectively; non-parametric Kolmogorov-Smirnov tests). The mapping between EPSP amplitude and short-term plasticity across the model inputs (Fig. $4 \mathrm{~A}, \mathrm{~B}$ ) followed the same relationship as observed in vitro: EPSP amplitudes $>2 \mathrm{mV}$ had significantly lower paired-pulse ratios (mean \pm s.d.: $0.82 \pm 0.08$ ) compared with EPSP amplitudes < $2 \mathrm{mV}$ (Fig $3 \mathrm{E}$; mean \pm s.d.: $0.92 \pm 0.20$; $p<0.0001$, parametric Welch's $t$ test), and due to the larger sample size compared to our in vitro data, there was a negative correlation between EPSP amplitude and paired-pulse ratio $(r=-0.26, p<0.0001$, $n=270$, non-parametric Spearman correlation coefficient). In accordance with electrophysiological recordings obtained from rodent sensory L2/3 in vivo (O'Connor et al., 2010), the firing rates of the inputs followed a lognormal distribution $\left(R^{2}=0.88\right.$ ) with a mean of $1.9 \pm 2.4 \mathrm{~Hz}$ (Fig. $4 \mathrm{C}$ ), the strong synaptic inputs had a mean firing rate of $6.4 \pm 4.1 \mathrm{~Hz}$ (maximum: $17.7 \mathrm{~Hz}$ ), and the weak synaptic inputs had a mean firing rate of $1.2 \pm 0.9 \mathrm{~Hz}$ (Fig. $3 \mathrm{G}$ ). The strong inputs exhibited the highest pairwise correlation coefficients (mean \pm s.d.: $0.24 \pm 0.09$; range: 0.11 to 0.45 ), while the weak inputs exhibited little correlation (mean \pm s.d.: $0.02 \pm 0.04$; range: -0.05 to 0.15 )( Fig. $3 \mathrm{~F}$ )(Cossell et al., 2015).

To examine information transfer between the synaptic inputs and the output firing pattern of the model neuron, we measured the Pearson correlation coefficient between each input spike train and the model neuron's output spike train. We further characterized the neuronal gain of the model cell by mapping its input-output relationship (i.e., the probability of spiking as a function of the number of coincident synaptic 
bioRxiv preprint doi: https://doi.org/10.1101/2021.12.21.473708; this version posted December 23, 2021. The copyright holder for this preprint (which was not certified by peer review) is the author/funder, who has granted bioRxiv a license to display the preprint in perpetuity. It is made available under aCC-BY-NC-ND 4.0 International license.

inputs). By selectively manipulating the relationship between synaptic strength, short-term plasticity, and temporal structure in the synaptic inputs, we then systematically characterized the contribution of each of these parameters on information transfer and neuronal gain. Each experiment was repeated for a total of 100 simulation runs; whereby for each iteration, we randomly re-generated a new set of 270 input spike trains.
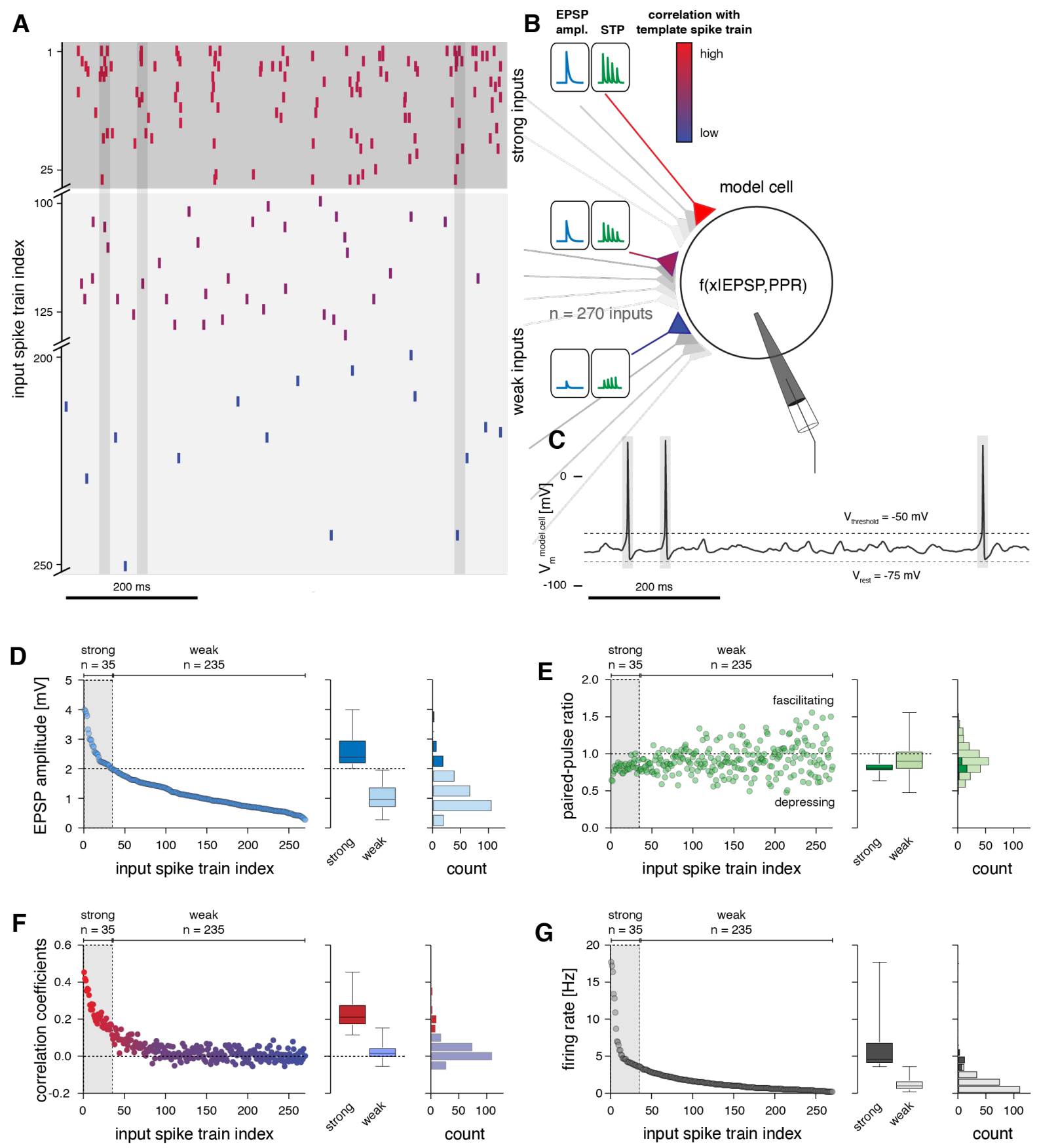

Figure 3. Default setup of the L2/3 neuron model.

A Example of input spike trains fed to the model cell. Strong inputs (top) fired with higher frequencies and temporal correlation (color coded), compared to weak inputs (bottom). Vertical grey bands indicate the resulting spike timing in the model cell (same as in C). Note that some weak inputs did not spike in the depicted $200 \mathrm{~ms}$ time window because of their low firing rates.

B Strong inputs were set to have larger EPSP amplitudes and corresponding short-term depression, while weak inputs were set to evoke smaller EPSPs and correspondingly weak net short-term plasticity, in accordance with our in vitro recordings. 
bioRxiv preprint doi: https://doi.org/10.1101/2021.12.21.473708; this version posted December 23, 2021. The copyright holder for this preprint (which was not certified by peer review) is the author/funder, who has granted bioRxiv a license to display the preprint in perpetuity. It is made available under aCC-BY-NC-ND 4.0 International license.

C Simulated membrane potential of model neuron following activation with the input spike trains shown in A.

D Left, EPSP amplitudes across the 270 input spike trains. Center, comparison of EPSP amplitudes between strong and weak inputs (median, $25-75 \%$ percentile, and ranges are indicated). Right, same data plotted as histogram.

E Left, 20 ms paired-pulse ratios across the 270 input spike trains. Center, comparison of paired-pulse ratios between strong and weak inputs (median, 25 - $75 \%$ percentile, and ranges are indicated). Right, same data plotted as histogram.

F Left, Pearson correlation coefficients of the 270 input spike trains with the template spike train that was used to generate the pairwise correlation structure (see Methods); color code as in A, B. Center, comparison of correlation with template spike train between strong and weak inputs (median, $25-75 \%$ percentile, and ranges are indicated). Right, same data plotted as histogram. G Left, firing rates of the 270 input spike trains. Center, comparison of firing rates between strong and weak inputs (median, 25 $-75 \%$ percentile, and ranges are indicated). Right, same data plotted as histogram.
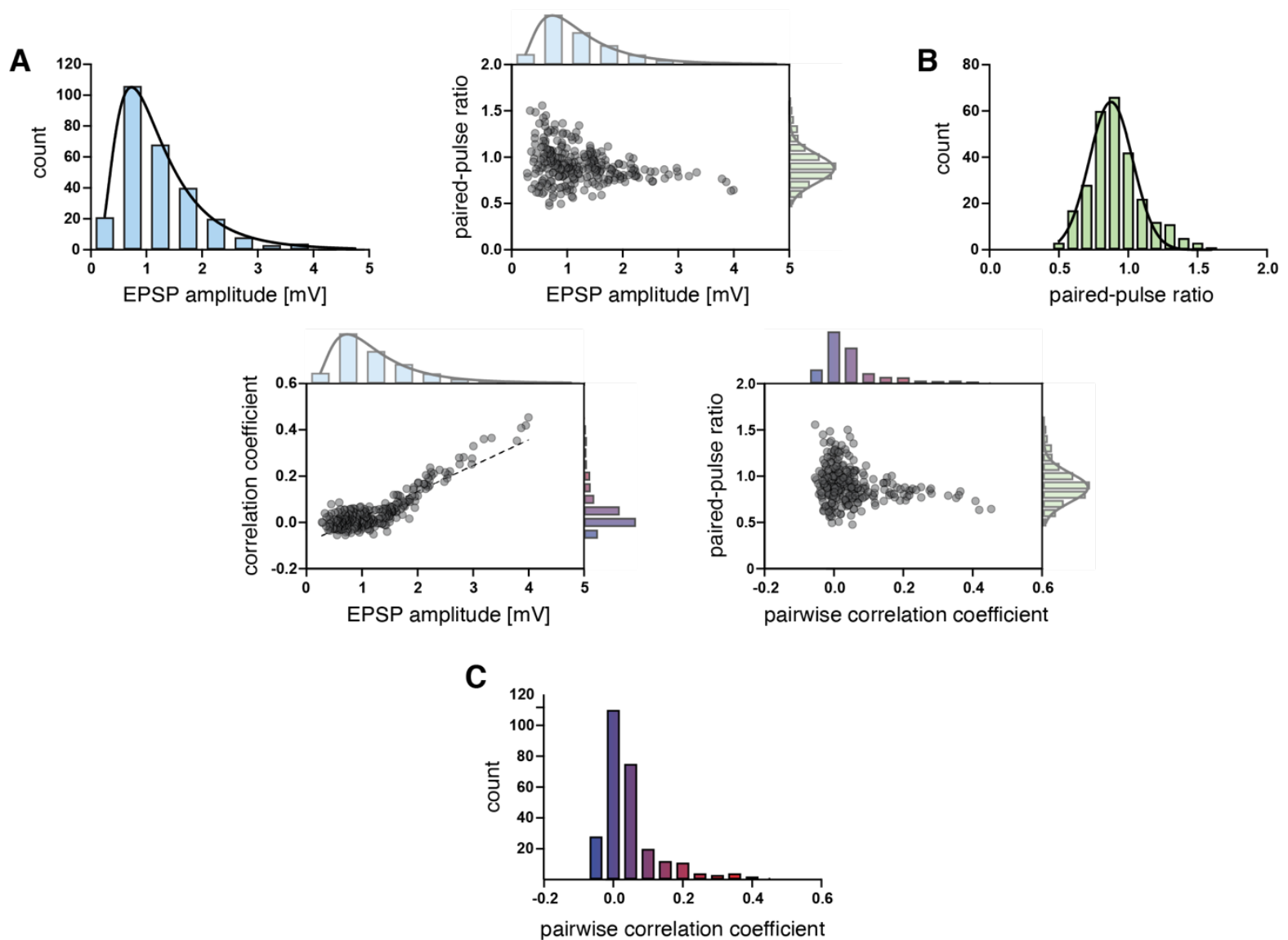

Figure 4. Mapping between synaptic strength, short-term plasticity, and correlation in input spike trains.

Figure shows distributions and mappings from a single, randomly selected run of the simulation.

A EPSP distribution for 270 inputs generated from our in vitro recordings.

B $20 \mathrm{~ms}$ paired-pulse ratio distribution for 270 inputs generated from our in vitro recordings.

C Pairwise-correlation coefficients for 270 inputs generated from in vivo data adopted from Cossell et al. (2015).

Interspersed scatter plots show respective pairwise mappings between the distributions in A-C. The relationship between parameter distributions reflected our in vitro data and in vivo data adopted from Cossell et al. (2015).

First, we ran the simulation in its default 'physiological' setup, i.e., with parameters and parametermappings as found in our in vitro recordings and published in vivo data (Fig. 3, 5). Critically, without further tuning, the model neuron reproduced key properties of rodent L2/3 pyramidal neurons in vivo. It generated output spike trains with an average firing rate of $4.81 \pm 0.71 \mathrm{~Hz}$ (Fig. $3 \mathrm{C}, 5 \mathrm{~F}$ ), which is in 
excellent agreement with experimental measurements of in vivo spike rates in mouse barrel cortex L2/3 (O'Connor et al., 2010). The average membrane voltage $\left(\mathrm{V}_{\mathrm{m}}\right)$ of the model neuron was $-65.93 \mathrm{mV} \pm$ $7.82 \mathrm{mV}$ (excluding action potentials) (Fig. $5 \mathrm{~B}, \mathrm{D}$ ), comparable to in vivo whole-cell recordings in mouse L2 (Jouhanneau et al., 2015). As expected, the strong synaptic inputs shared the highest Pearson correlation coefficients (mean \pm s.d.: $0.11 \pm 0.038$; range: 0.064 to 0.20 ) with the resulting output spike train of the model neuron (Cossell et al., 2015), while the weak inputs displayed correlation coefficients one order of magnitude smaller (mean \pm s.d.: $0.012 \pm 0.013$; range: -0.0041 to 0.065 ) (Fig. $5 \mathrm{E}$ ). Across all inputs, spike trains with decreasing intrinsic correlation, smaller EPSP amplitudes, and lower spike rates displayed increasingly lower correlation coefficients with the output spike train (Fig. $5 \mathrm{E}$ ). We confirmed that the Pearson correlation coefficients indeed detected correlations in spike timing rather than in firing rates by randomizing the output spike times following a random Poisson process while keeping the output firing rate identical. Reassuringly, the correlations between all inputs and the output spike train then dropped to $-0.0005 \pm 0.0032$ (not shown).

\section{Synaptic background activity enhances information transfer of strong inputs}

We probed the relative influence of the strong versus weak synaptic inputs on the output spiking of our model cell. Critically, when we removed the weak inputs (Fig. 5 A, B), the mean correlation between the strong inputs and the output spike train was reduced to $0.068 \pm 0.019$ (range: 0.034 to 0.10 ) (Fig. 5 E). The output firing rate of the model neuron dropped to $1.26 \pm 0.34 \mathrm{~Hz}$ (Fig. $5 \mathrm{~F}$ ) and its average $\mathrm{V}_{\mathrm{m}}$ was hyperpolarized to $-68.39 \mathrm{mV} \pm 4.57 \mathrm{mV}$ (excluding action potentials). Despite the 2 -fold drop in information transfer of the strong synaptic inputs, those inputs with the highest intrinsic correlation and synaptic strength still maintained the highest correlation with output spiking (Fig. 5 E). Removal of weak inputs also resulted in a steeper slope of the input-output curve (Fig. $5 \mathrm{G}$ ), confirming that synaptic 'background noise' has a divisive effect on neuronal gain. This noise broadens a neuron's sensitivity to the range of temporal correlations in input spike trains by increasing the time window over which coincident inputs can be integrated to evoke spiking, a finding in agreement with previous studies (Silver, 2010).

Conversely, when we removed the strong synaptic inputs from the simulation (Fig. $5 \mathrm{C}$ ), uncorrelated activity provided by the weak inputs was by itself unable to drive the postsynaptic neuron above spiking threshold and the output firing rate dropped to $0.045 \pm 0.068 \mathrm{~Hz}$ (Fig. $5 \mathrm{~F}$ ). This is because the 235 weak inputs fired at an average frequency of $1.2 \pm 0.9 \mathrm{~Hz}$ with mean EPSP amplitudes of 1.03 $\pm 0.42 \mathrm{mV}$, which resulted in a mean membrane potential of $67.68 \pm 1.67 \mathrm{mV}$ (excluding action potentials) that rarely crossed the spike threshold (Fig. $5 \mathrm{D}$ ). Thus, uncorrelated activity of weak synapses alone was incapable of evoking spikes and did not transfer information encoded in its own spike trains (Fig. 5 E). Importantly, however, it had a powerful computational effect on neuronal activity because it enhanced information transfer of the strong, correlated inputs by a factor of 2 . 
A

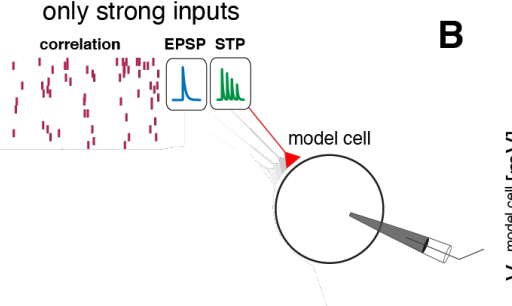

C

only weak inputs

correlation EPSP STP

E

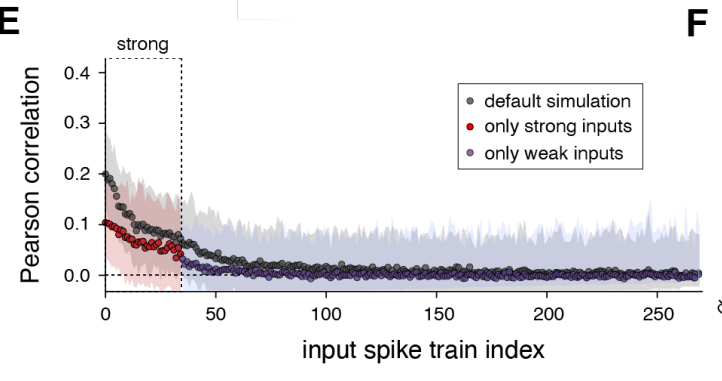

$\mathbf{F}$
D

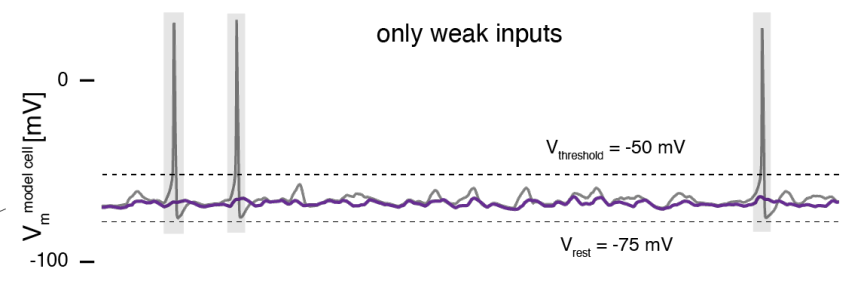

only strong inputs
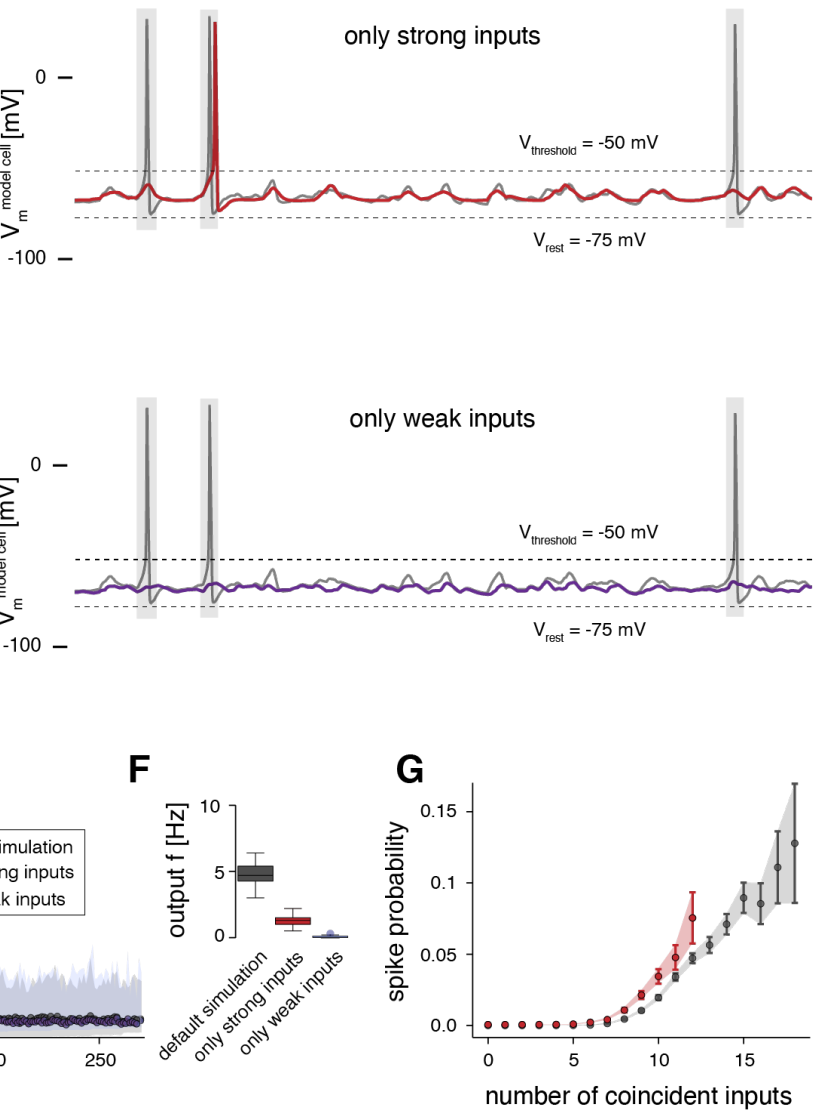

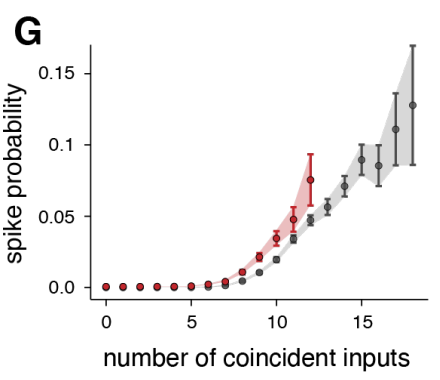

Figure 5. Uncorrelated activity from weak inputs enhances information transfer of strong synaptic inputs

A Schematic of model setup with weak inputs removed.

B Example spike train of the model cell in its default setup (grey) and when weak inputs are removed (red).

C Schematic of model setup with strong inputs removed.

D Example spike train of the model cell in its default setup (grey) and when strong inputs are removed (purple).

E Pearson correlation coefficients of the 270 input spike trains with the output spike train of the model cell. Results of three model setups are shown: default simulation (all inputs, as in Fig. 3) and setups introduced in A, C. Shaded regions, $95 \%$ confidence bounds for correlation coefficients obtained from 100 runs of the simulation.

F Respective firing rates of model cell for the three model setups.

G Probability of output spiking as a function of coincident spikes across all input spike trains. (Note that the maximum number of coincident inputs within the 20 ms measurement window was 12 when only strong inputs were included, thus determining the maximum $x$-value for the red curve.)

\section{Output spiking requires correlation and high firing rates of strong inputs}

Next, we decoupled the high temporal correlation and high firing rates of strong inputs from their larger synaptic strengths by randomly assigning the EPSP amplitudes and their corresponding short-term plasticity properties across the input spike trains (Fig. 6 A). Note that the original coupling between EPSP amplitude and short-term plasticity was maintained in this experiment, i.e., synapses with larger EPSPs still exhibited depression and synapses with smaller EPSPs exhibited facilitation.

When the model was set up in this manner, the firing rate of the output neuron decreased to $1.11 \pm 0.68 \mathrm{~Hz}$ (Fig. $6 \mathrm{~B}, \mathrm{D}$ ). Critically, inputs with higher temporal correlation and higher firing rates still 
bioRxiv preprint doi: https://doi.org/10.1101/2021.12.21.473708; this version posted December 23, 2021. The copyright holder for this preprint (which was not certified by peer review) is the author/funder, who has granted bioRxiv a license to display the preprint in perpetuity. It is made available under aCC-BY-NC-ND 4.0 International license.

contributed more strongly to the firing of the model neuron (Pearson correlation mean \pm s.d.: $0.056 \pm$ 0.016 ; range: 0.035 to 0.095 ) compared to inputs with lower temporal correlations and lower firing rates (mean \pm s.d.: $0.009 \pm 0.008$; range: -0.004 to 0.042 ) (Fig. 6 C). This means that synaptic strength by itself did not determine which inputs transmitted the most information to the spike train of the output neuron. Instead, in our simulation, the combination of high temporal correlation and elevated firing rates of strong synaptic inputs was the primary determinant for evoking correlated spiking in the output neuron. However, matching larger EPSP amplitudes to inputs that fired with high temporal correlation and high firing rates (i.e., our default setup), as observed for the strong synaptic inputs in vivo (Cossell et al., 2015), increased their correlation with the spike train of the model neuron by a factor of 2 and enhanced their information transfer (Fig. 6 C). Decoupling the large EPSP amplitudes from the correlated inputs (by shuffling EPSP amplitudes amongst all input spike trains) furthermore resulted in a flatter slope of the model's input-output curve and a reduced responsiveness to coincident inputs (maximum spike probability $\left(P_{\max }\right)$ of 0.15 ; Fig $6 \mathrm{E}$ ). This suggests that assigning the largest EPSP amplitudes to those inputs that fired at high temporal correlation has a multiplicative effect on neuronal gain, leading to signal amplification as a mechanism to increase efficient information transmission of strong inputs (Silver, 2010).
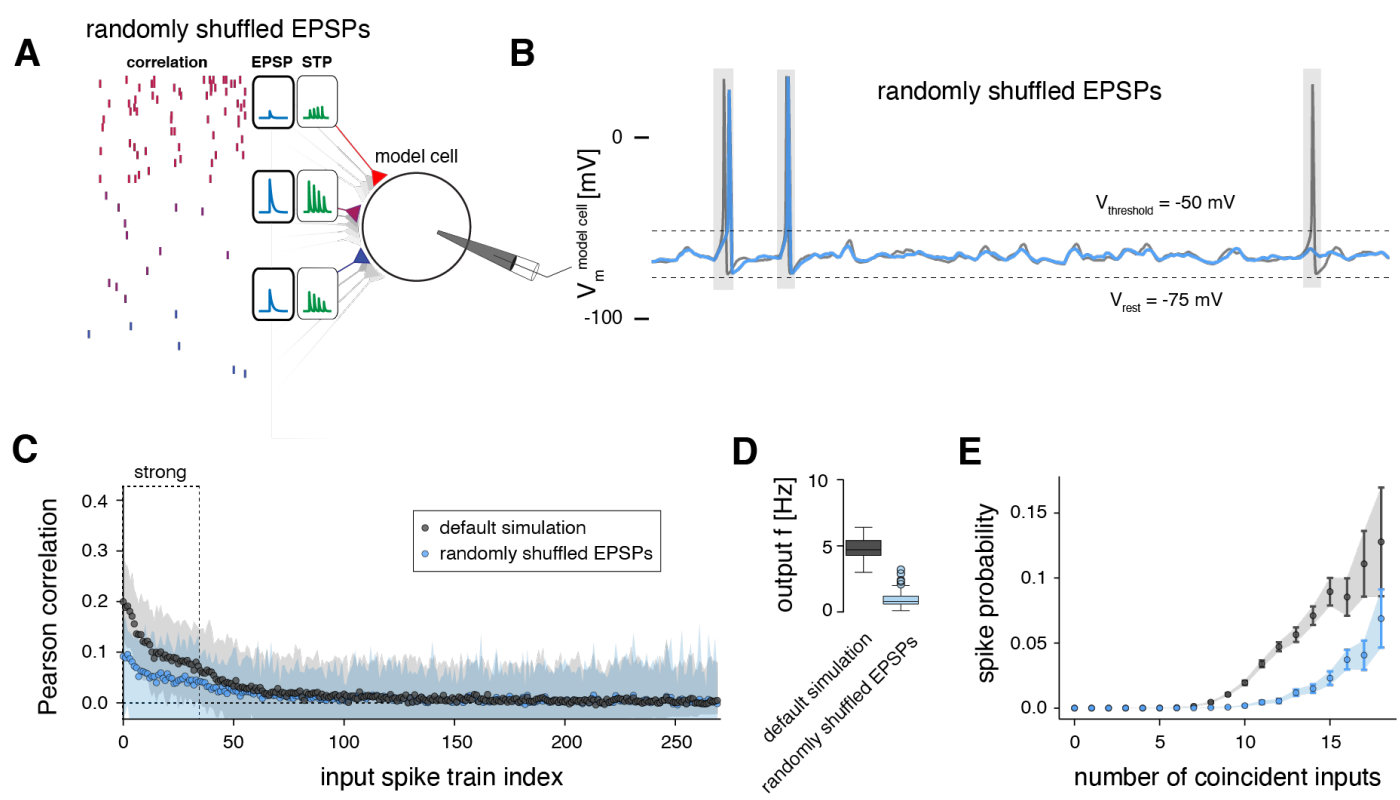

Figure 6. Temporal correlation and firing rates primarily determine output spiking, synapse strength enhances response. A Schematic of model setup with shuffled EPSP amplitudes; note that the relationship of EPSP amplitude and short-term plasticity was maintained.

B Example spike train of the model cell in its default setup (grey) and with shuffled EPSP amplitudes (blue).

C Pearson correlation coefficients of the 270 input spike trains with the output spike train of the model cell. Results of two model setups are shown: default simulation (as in Fig. 3) and setup introduced in A. Shaded regions, $95 \%$ confidence bounds for correlation coefficients obtained from 100 runs of the simulation.

D Respective firing rates of model cell for the two model setups.

E Probability of output spiking as a function of coincident spikes across all input spike trains. 


\section{Short-term plasticity balances the computational effects of strong and weak inputs}

Next, we removed the short-term plasticity mechanism from all synapses, such that they exhibited paired-pulse ratios of 1 for all inter-spike-interval durations, i.e., synaptic strength remained static during repeated stimulation (Fig. 7 A, B).

When running the simulation in this setup, the model neuron fired at $17.4 \pm 0.63 \mathrm{~Hz}$, which was an even higher frequency than exhibited by those input spike trains with the highest firing rates (Fig. 7 F). At the same time, the mean correlation coefficient of the strong inputs with the output spike train of the model neuron had doubled to $0.20 \pm 0.082$, with the largest values exceeding 0.4 (Fig. $7 \mathrm{E}$ ). Also the correlation coefficients of the weak inputs with the output spike train had increased to $0.018 \pm 0.023$ (Fig. 7 E). Notably, because the weak synaptic inputs had been only mildly depressing on average in our default setup (Fig. $3 \mathrm{E}$ ), removing their short-term plasticity mechanism should only have a small net boosting effect on their total excitatory drive. To confirm this, we additionally removed the weak inputs from the model entirely (Fig. 7 C, D) and found that this indeed had no significant effect on the correlation coefficients between the strong inputs and the resulting spike train of the model neuron (Fig. $7 \mathrm{E}$ ) nor on the output firing rate of the model cell (Fig. $7 \mathrm{~F}$ ). Thus, after removing short-term plasticity, the computational effect of the weak inputs in maximizing information transfer of strong inputs had become entirely redundant. In this regime, the strong synapses alone could determine the spiking properties of the model neuron.

Furthermore, the slopes of the input-output curves were markedly steeper when the short-term plasticity mechanism was removed and when the weak inputs were removed in addition (Fig. $7 \mathrm{G}$ ), which confirms that short-term depression of strong inputs has a divisive impact on neuronal gain (Abbott et al., 1997; Rothman et al., 2009), therefore broadening the neuron's responsiveness to temporal correlations in input spike trains (Silver, 2010). 
A no STP, all inputs
correlation EPSP STP

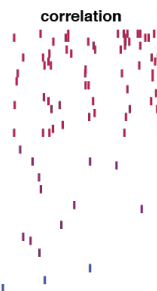
EPSP STP

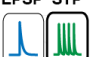

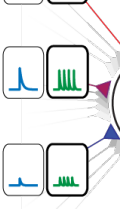

',

C

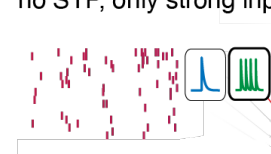

B no STP, all inputs

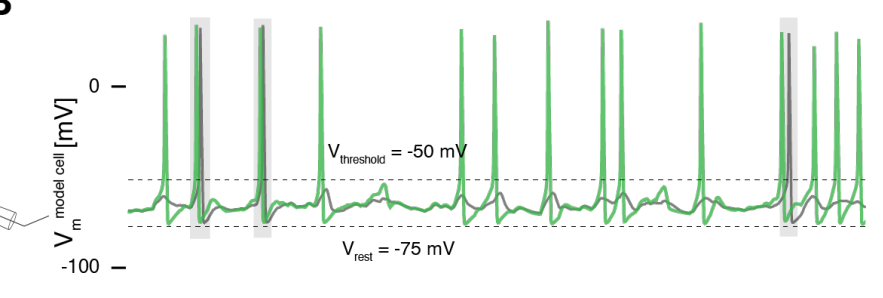

D no STP, only strong inputs
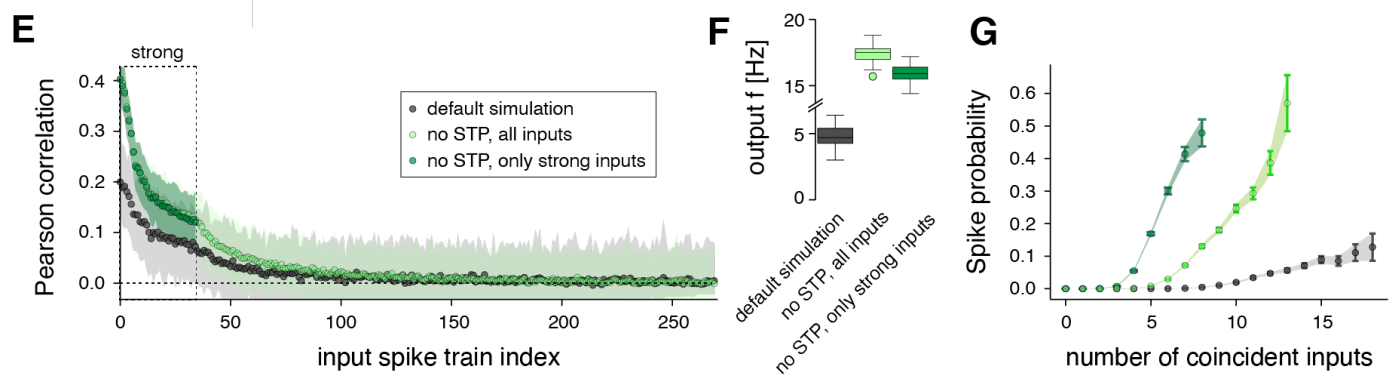

Figure 7. Short-term plasticity balances the computational effects of strong and weak inputs

A Schematic of model setup with short-term plasticity mechanisms removed; note that all spike trains exhibit a paired-pulse ratio of 1.

B Example spike train of the model cell in its default setup (grey) and when short-term plasticity mechanisms are removed (light green).

C Schematic of model setup with short-term plasticity mechanisms removed and the weak inputs removed in addition.

D Example spike train of the model cell in its default setup (grey) and when short-term plasticity mechanisms and weak inputs are removed (dark green).

E Pearson correlation coefficients of the 270 input spike trains with the output spike train of the model cell. Results of three model setups are shown: default simulation (as in Fig. 3) and setups introduced in A, C. Shaded regions, $95 \%$ confidence bounds for correlation coefficients obtained from 100 runs of the simulation.

F Respective firing rates of model cell for the three model setups.

G Probability of output spiking as a function of coincident spikes across all input spike trains. 


\section{Discussion}

We combined experimental work and computational modeling to investigate how the spiking responses of $L 2 / 3$ pyramidal neurons are shaped by the complex parameter-space of temporal structure within synaptic inputs, synaptic strength, and short-term plasticity.

As a first step, we mapped experimentally the distribution of synaptic strength and short-term plasticity in barrel cortex L2/3. We found that short-term plasticity follows a symmetrical distribution with large variance and is mildly depressing on average. Interestingly, synaptic strength and short-term plasticity were only weakly negatively correlated across our large dataset. Instead, their relationship was well-captured by the simple rule that synaptic connections with EPSP amplitudes below $2 \mathrm{mV}$ span the full range of depression and facilitation and exhibit no pronounced average short-term plasticity. By contrast, connections with EPSP amplitudes above $2 \mathrm{mV}$ are exclusively depressing, which raises the intriguing question of what the computational role is for depression of strong synapses.

Our computational model of a L2/3 neuron suggested that the ability of the strong synaptic inputs to evoke spiking in postsynaptic cells relies predominantly on their high temporal correlation and high firing rates, as well as on synaptic background activity from the numerous weak synapses, and not primarily on their synaptic strength. Pairing those driving, 'co-tuned' synaptic connections with strong synaptic weights, however, as has been reported for rodent V1 (Cossell et al., 2015), does amplify their ability to transmit information to the output spike train.

\section{Short-term plasticity in barrel cortex L2/3}

While mild average depression in rodent barrel cortex $L 2 / 3$ is in agreement with previous reports (Reyes and Sakmann, 1999; Feldmeyer et al., 2006), other studies have found excitatory L2/3 synapses in sensory areas to be moderately facilitating on average (Jouhanneau et al., 2015; Lefort and Petersen, 2017; Seeman et al., 2018). Intriguingly, Jouhanneau et al. (2015) and Lefort and Petersen (2017) used paired recordings in layer 2 (L2). While Seeman et al. (2018) conducted paired recordings across the entire $L 2 / 3$, they reported only mild average facilitation with large overall heterogeneity.

We recorded from neuronal somata in superficial L2/3, likely corresponding to the layer investigated by Jouhanneau et al. (2015) and Lefort and Petersen (2017), but stimulated axons of passage across the entire depth of $L 2 / 3$. Thus, differences between these datasets may indicate differences in synaptic properties between L2 recurrent connections (Jouhanneau et al., 2015; Lefort and Petersen, 2017) and the L3 -> L2 pathway. This is in line with a growing body of literature describing structural (Karimi et al., 2020) and functional (Crochet et al., 2011; Petersen and Crochet, 2013) differences between the neuronal circuits in L2 and L3 and further supports the notion that L2 and L3, which are routinely considered to constitute a single computational entity, may in fact possess different computational properties (Petersen and Crochet, 2013; Karimi et al., 2020).

\section{No evidence for a statistical bias of synaptic innervation on L2/3 neurons}

Interestingly, we found no statistical bias of synaptic strength or short-term plasticity of synaptic connections formed with the same pyramidal neurons in L2/3. Instead, our data suggest that synaptic inputs formed with a given L2/3 neuron are not markedly correlated, but that their strengths and short- 
term plasticity instead follow the same distribution as that of all synaptic connections across the neuropil. Such statistical biases have been hypothesized to explain lognormal firing rate distributions in cortex (Koulakov et al., 2009) and have been proposed as a potential mechanism for endowing neurons with high-pass or low-pass filter properties that may underlie integration and differential activation (Lisman, 1997; Fortune and Rose, 2001; Abbott and Regehr, 2004). Importantly, by demonstrating the absence of such systematic biases on the single-cell level, our experimental results provide a critical biological constraint for theoretical models of how these particular computations may arise in L2/3.

Because we have characterized synaptic strength and short-term plasticity through somatic whole-cell recordings, we cannot exclude the intriguing possibility that statistical biases of synaptic innervation may exist on the level of dendritic branches, in which case such computations may be implemented on a sub-cellular level. Further experiments will be necessary to investigate this possibility.

\section{L2/3 neuron model reproduces key computation properties of cortical circuits}

To address the computational role of depression of strong connections and to investigate how synaptic strength, short-term plasticity, and temporal properties in presynaptic spike trains within the L2/3 circuitry shape the firing properties of neurons, we generated a simplified model of a L2/3 pyramidal neuron and systematically manipulated these parameters in our simulation. The synaptic inputs to the model neuron were constrained by physiological data obtained from our own in vitro recordings and with in vivo data adopted from the literature (O'Connor et al., 2010; Cossell et al., 2015). To focus our study on the L2/3 circuitry, we constrained the model neuron to receive synaptic connections only from other L2/3 neurons, i.e., connections from layer 4 and the deep layers were not modeled, such that it was not necessary to include inhibitory synapses in the simulation to balance excitation. Reassuringly, without further parameter tuning, the model exhibited key computational properties of cortical neurons that have been characterized in experiments and simulations before: the model cell produced sparse firing at around $5 \mathrm{~Hz}$, which is in excellent agreement with the average spike rate reported for mouse barrel cortex L2/3 (O'Connor et al., 2010), and its output spike train exhibited the highest temporal correlation with the strong synaptic inputs (Cossell et al., 2015). In addition, our simulations could reproduce the effects of multiplicative gain modulation through synaptic background activity (Salinas and Sejnowski, 2001; Chance et al., 2002) and through short-term depression of strong synapses (Abbott et al., 1997; Rothman et al., 2009).

We found that synaptic background activity carried through the weak synapses contributed critically to information transfer of strong inputs through a stochastic resonance-type effect (Faisal et al., 2008): while being incapable of evoking spiking by itself, the weak inputs enabled the model neuron to operate in a regime in which the cell became sensitive and responsive to coincident strong inputs (Bulsara et al., 1991; Hô and Destexhe, 2000; Chapeau-Blondeau and Rousseau, 2002; London et al., 2002; McDonnell and Abbott, 2009; Durand et al., 2013). Even then, the high firing rates and the synchronous activity of multiple strong synapses were needed to evoke spiking in the model neuron (Bruno and Sakmann, 2006; Banitt et al., 2007; Wang et al., 2010; Schoonover et al., 2014; Martin and Schröder, 2016). Notably, synaptic strength alone did not determine which presynaptic cells could evoke spikes (Scholl et al., 2020). 


\section{Short-term depression balances the synaptic drive of strong inputs}

The computational role of the relationship between short-term plasticity and synaptic strength has not been addressed in detail in studies of cortical processing. Interestingly, the pronounced short-term depression we observed for synaptic connections eliciting large EPSPs in vitro proved necessary to counterbalance the high firing rates, high temporal correlations, and large EPSP amplitudes of strong inputs during ongoing stimulation and was critical for maintaining the responsiveness of the postsynaptic neuron towards input spike trains with the highest temporal correlation. This suggests that short-term depression could act as one of the mechanisms that prevent runaway excitation in the recurrent $L 2 / 3$ circuitry.

\section{A framework for orientation tuning in columnar and 'salt-and-pepper' cortices}

The notion that the minority of strong synaptic inputs determines the response properties of cortical neurons (Cossell et al., 2015; Znamenskiy et al., 2018; Goetz et al., 2021) has recently been challenged by apparently conflicting findings made in V1 of the ferret (Scholl et al., 2020). In mouse V1, neurons with the most similar receptive field properties in vivo also formed the strongest synaptic connections with each other, as assessed in vitro (Cossell et al., 2015). By contrast, the response selectivity of neurons in ferret $\mathrm{V} 1$ in vivo was shown to be determined by the cumulative weight of all driving synapses - weak and strong. Intriguingly, the response selectivity could not be predicted from the tuning of strong synapses alone (Scholl et al., 2020).

Our result that spiking in the model neuron was driven predominantly by high temporal input correlation and high firing rates, while synaptic strength further enhanced information transfer of these driving inputs may provide a framework to reconcile these apparently contradictory findings. In the columnar V1 of carnivores (Hubel and Wiesel, 1962), presentation of simple visual stimuli activates populations of neighboring neurons within the same orientation column (Ohki et al., 2005). The axons of pyramidal cells in the superficial layers of $\mathrm{V} 1$ form a primary cluster of synaptic boutons around their own somata (Martin et al., 2014). Thus, unlike in rodents, these neurons are excited by many neighboring neurons with the same orientation tuning and ocular dominance. Therefore, 'columnar' orientation maps, which are found in visual areas of higher mammals (Gilbert and Wiesel, 1989; Malach et al., 1994; Bosking et al., 1997; Sincich and Blasdel, 2001) may provide the basis for the "strength by numbers" necessary to generate tuned responses (Scholl et al., 2020), without the additional requirement of stronger synapses between co-tuned neurons. Our finding that the high temporal correlation and firing rates of strong inputs, and not their larger synaptic strength primarily drive spiking supports this idea and is consistent with the observation that spikes in cat V1 are phase-locked with the local field potential, which reflects synchrony within local neuronal populations (Martin and Schröder, 2016).

By contrast, the 'salt-and-pepper' organization of rodent V1 (Girman et al., 1999), means that oriented stimuli activate a spatially diffuse network (Ohki et al., 2005). Therefore, neurons may receive fewer synaptic connections overall from similarly tuned cells and temporal correlation and firing rates alone may be insufficient to achieve orientation tuning. Our observation that pairing large EPSP amplitudes with correlated input spike trains further enhances the capacity of driving inputs to transmit 
information suggests that this predicted 'lack of strength by numbers' in rodent $\mathrm{V} 1$ may be compensated for by stronger synapses between similarly tuned neurons (Cossell et al., 2015). This, however, leads to the prediction that in mouse $\mathrm{V} 1$, the temporal structure in input spike trains from similarly tuned neurons also plays a key role in generating orientation tuning in vivo, a prediction that could be tested experimentally.

Our results provide a holistic framework of how cortical neurons could utilize synergies between the biophysical properties of chemical synapses, the temporal structure of input spike trains, and 'noise' in neuronal networks for efficient computation. 


\section{Methods}

\section{Animals}

Cortical slices were obtained from 13 male B6/C57 mice between 22 and 29 postnatal days of age under the license of Kevan A.C. Martin (Institute of Neuroinformatics, University of Zurich \& ETH Zurich, Zurich, Switzerland). Animal handling and experimental protocols were approved by the Cantonal Veterinary Office, Zurich, Switzerland.

\section{Slice preparation}

Animals were anesthetized with isoflurane, decapitated, and their brains were removed quickly and immersed in ice-cold slicing artificial cerebrospinal fluid (ACSF, containing, in mM: $87 \mathrm{NaCl}, 75$ sucrose, $26 \mathrm{NaHCO}_{3}, 10$ glucose, $7 \mathrm{MgSO}_{4}, 2.5 \mathrm{KCl}, 1 \mathrm{NaH}_{2} \mathrm{PO}_{4}$, and $0.5 \mathrm{CaCl}_{2}$, continuously oxygenated with $95 \% \mathrm{O}_{2}, 5 \% \mathrm{CO}_{2}$ ). Coronal slices containing the barrel cortex were cut at a thickness of $300 \mu \mathrm{m}$ on a vibratome and transferred to a chamber containing recoding ACSF (containing, in mM: $119 \mathrm{NaCl}, 26$ $\mathrm{NaHCO}_{3}, 10$ glucose, $1.3 \mathrm{MgSO}_{4}, 2.5 \mathrm{KCl}, 1.25 \mathrm{NaH}_{2} \mathrm{PO}_{4}$, and $2.5 \mathrm{CaCl}_{2}$, continuously oxygenated with $95 \% \mathrm{O}_{2}, 5 \% \mathrm{CO}_{2}$ ). The slices were kept in recording ACSF at room temperate until the recordings.

\section{Electrophysiology}

Patch pipettes (pipette resistance: 5-7 M $\Omega$, pipette tip diameter: $2 \mu \mathrm{m}$ ) were pulled from borosilicate glass using a P-97 puller (Sutter Instruments) and filled with intracellular solution (containing in $\mathrm{mM}$ : $105 \mathrm{~K}$-gluconate, $20 \mathrm{KCl}, 10 \mathrm{Na}$-phosphocreatine, $2 \mathrm{Mg}$-ATP, $2 \mathrm{Na}-\mathrm{ATP}, 0.3 \mathrm{GTP}$, and $10 \mathrm{HEPES}, \mathrm{pH}$ was set to 7.2 with $\mathrm{KOH})$. Biocytin $(0.5 \%)$ was added to the intracellular solution to stain the recorded neurons. Whole-cell patch-clamp recordings were obtained at $34-36{ }^{\circ} \mathrm{C}$ from visually identified $\mathrm{L} 2 / 3$ neurons in barrel cortex under an Olympus BX61W1 microscope equipped with infrared differentialinterference contrast optics and a 10x and a 60x water-immersion objective. Data were acquired with a Multiclamp 700A amplifier (Axon Instruments), sampled at $10 \mathrm{kHz}$, filtered at $3 \mathrm{kHz}$ (Digidata 1322A, Axon Instruments) and monitored with the software pClamp (Molecular Devices).

Following break-in, the access resistance was typically in the range of 15-30 $\mathrm{M} \Omega$ and recordings with an access resistance $>30 \mathrm{M} \Omega$ were discarded. The bridge potential was compensated and liquidjunction potential was not corrected. Membrane potentials $\left(V_{m}\right)$ after break-in ranged from -85 to -70 $\mathrm{mV}$. If the membrane potential drifted during recordings, a holding current was injected to keep the membrane at its initial resting potential, which was rarely necessary.

After establishing whole-cell recordings, we identified presynaptic axons forming synapses with the recorded cells by carefully moving a monopolar extracellular stimulation electrode (filled with ACSF) through $L 2 / 3$ at an oblique angle and delivering repeated 0.1 ms current pulses of 10-12 $\mu \mathrm{A}$ amplitude using an A360 stimulator (World Precision Instruments) until an EPSP was detected in the patched neuron. Synaptic connections were typically detected when the stimulation electrode was located 20$400 \mu \mathrm{m}$ distant from the soma of the recorded cell. To achieve minimal stimulation of single axons, we then decreased the stimulation amplitude until the EPSP was not elicited anymore and subsequently increased the stimulation amplitude until a clear EPSP was seen in a high fraction of trials. The final 
stimulation amplitude was set to this level (typically 5-16 $\mu \mathrm{A}$ ). As an additional control to ensure we stimulated single axon fibers, we recorded only synaptic connections for which the EPSPs were elicited in an all-or-none manner and which showed little or no variability in EPSP latency or shape from trial to trial. We then performed $20 \mathrm{~ms}$ paired-pulse stimulation at a low frequency $(0.2 \mathrm{~Hz})$ for at least 30 sweeps. In some cases, the stimulation electrode position was slightly readjusted in the $z$ dimension to correct for drift. Data were only used when the EPSP amplitude at the end the recordings had the identical latency and shape compared to the first evoked minimal stimulation EPSPs. Following the minimal stimulation protocol, we carefully moved the extracellular stimulation electrode to other locations in the L2/3 neuropil to identify different axon fibers forming synapses with the same recorded neuron. Great care was taken not to record from the same stimulation location multiple times, and synaptic connections were only included when their location of stimulation was $>50 \mu \mathrm{m}$ away from all previous stimulation locations, as assessed in 10x overview images during recordings. At the end of each experiment, we injected current steps into each neuron to characterize its firing pattern as regularspiking (i.e., putatively excitatory/ pyramidal neuron) or fast-spiking (putatively inhibitory/ interneuron).

\section{Histology}

After recordings, slices were immediately fixed in 15\% picric acid, $4 \%$ paraformaldehyde, and $0.5 \%$ glutaraldehyde in $0.1 \mathrm{M}$ phosphate buffer $(\mathrm{PB})$ overnight. Fixed slices were then washed in $\mathrm{PB}$, incubated in an ascending sucrose ladder for cryoprotection, quickly frozen in liquid nitrogen, and treated in 3\% hydrogen peroxide and 10\% methanol in phosphate-buffered saline (PBS) to quench endogenous peroxidases. After washing in PBS and tris-buffered saline (TBS), the slices were treated with the Vectastain ABC Kit (Vector Laboratories, catalog \# PK-6100, RRID: AB_2336819) in TBS at 4 ${ }^{\circ} \mathrm{C}$ overnight. Following washing in TBS, biocytin was visualized using nickel-diaminobenzidine (Ni$\mathrm{DAB}$ ) tetrahydrochloride and hydrogen peroxide treatment, followed by a series of washes in PB to terminate the reaction. Sections were then embedded in Mowiol (Sigma Aldrich) and cover-slipped. Zstacks of the recovered neurons were imaged under an Olympus BX61 microscope to cross-check the previously determined electrophysiological cell type with anatomy. Pyramidal cells and interneurons were identified on the basis of their dendrite morphology (e.g., spiny dendrites versus smooth dendrites, respectively) and corresponded with the previously recorded regular-spiking firing pattern and fastspiking firing patterns, respectively.

\section{Analysis of electrophysiological data}

We analyzed each postsynaptic potential evoked with paired-pulse stimulation with Stimfit (Guzman et al., 2014) and measured its peak amplitude, coefficient of variation, onset latency (i.e., the time from the onset of the extracellular stimulation artifact to the onset of the evoked postsynaptic potential) and $10 \%-90 \%$ rise time. The EPSP was defined as the postsynaptic potential evoked by the first pulse of the paired-pulse paradigm, i.e., before STP took place. The paired-pulse ratio was defined as the peak amplitude of the second evoked postsynaptic potential divided by the peak amplitude of the first evoked postsynaptic potential (i.e., the EPSP). Further statistical analyses were done in Matlab (MathWorks) and Prism (GraphPad). 
To obtain an unbiased population distribution for a given experiment, we excluded all afferent synaptic connections formed with the postsynaptic neuron in that experiment, but otherwise included all other connections recorded in regular spiking neurons. The cell distribution for a given experiment included all afferent synaptic connections formed with the postsynaptic neuron in that experiment.

We conducted a post-hoc Monte-Carlo power analysis to estimate which effect sizes (i.e., systematic differences between mean EPSP amplitudes or mean paired-pulse ratios between the cell distribution and the population distribution) were detectable given the sample sizes in our dataset. We did this for each experiment individually by bootstrapping new cell distributions with systematically different means and then performing Kolmogorov-Smirnov tests against the population distribution.

Specifically, for the power analysis for paired-pulse ratios, we first formalized the paired-pulse ratio population distribution for each experiment as a normal distribution with the same mean and standard deviation as the experimentally observed paired-pulse ratio population distribution for that experiment. To test which effect sizes were detectable, we then formalized a range of possible underlying generator distributions for the paired-pulse ratio cell distribution for that experiment by varying the mean of the population distribution in steps of \pm 0.1 units. By doing so, we designed a range of generator distribution for the paired-pulse ratio cell distribution with systematically different means. For each one of these cell generator distributions, we then drew the same number of random samples that were present in the experimentally observed cell distribution (i.e., between 5 and 8 ) and ran a Kolmogorov-Smirnov tests against a random sample drawn from the formalized population distribution (containing the same number of entries as the population distribution for that experiment). This analysis was repeated 10,000 times for each cell generator distribution and the statistical power for detecting an effect of a certain size (i.e., the systematic difference in the means between the underlying cell generator distribution and the population distribution) was defined as the fraction of trials that yielded a significant $p$-value $(\alpha=0.05)$, see Results. The power analysis for EPSP amplitudes was done in an analogous fashion with the only exception that lognormal distributions were used instead of normal distributions, in accordance with our results.

Because our dataset contained 8 experiments for which at least 5 afferent connections were mapped, there were 8 chances for detecting a significant difference between a cell and the population distribution across our experimental series. Thus, a simple binomial model can be used to ask: which systematic difference in paired-pulse ratios should have been observed in at least one of these 8 experiments at the $95 \%$ significance level? To answer this, we computed the probability density functions for obtaining zero as a realization (i.e., the likelihood of observing no significant difference across any of the 8 experiments) of simple binomial functions with $N=8$ (i.e., the number of our independent experiments) and $\mathrm{P}=$ the average probability of observing a given effect size in a single experiment (as derived above, see Results). We then repeated these analyses in an analogous fashion for the EPSP amplitude distributions. 


\section{Conductance-based model of L2/3 neuron}

We generated a simplified two-compartment, conductance-based model (Pinsky and Rinsky, 1994; Mainen and Sejnowski, 1996; Larkum, 2004; Yi et al., 2017) of a L2/3 pyramidal neuron in the NEURON software (Hines and Carnevale, 1997). The model neuron consisted of an active soma (diameter of 20 $\mu \mathrm{m})$ with a Hodgkin-Huxley spiking mechanism and a passive dendrite receiving all synaptic inputs (diameter of $2 \mu \mathrm{m}$; length of $100 \mu \mathrm{m}$ ). We set up the model in accordance with experimentally measured passive electrical properties of barrel cortex pyramidal cells, previous models of $L 2 / 3$ neurons, and our own experimental data. Parameters were chosen in accordance with previous literature such that somatic EPSPs elicited by synapses placed on the dendrite of the neuron model reproduced the same distribution that we measured experimentally with minimal stimulation in slices.

Because the exact ion-channel compositions for $L 2 / 3$ neurons are not well established, passive biophysical parameters are routinely modelled as being homogenously distributed in models of L2/3 neurons (Branco et al., 2010; Smith et al., 2013; Ferrarese et al., 2018). It has been determined experimentally that the specific axial resistance $\left(R_{\mathrm{i}}\right)$ of pyramidal neurons ranges between $70 \mathrm{Ohm} \mathrm{cm}$ to $100 \mathrm{Ohm} \mathrm{cm}$ (Stuart and Spruston, 1998), we set $R_{\mathrm{i}}$ of the dendrite to $100 \Omega \mathrm{cm}$ (Wang et al., 2010) to account for the shorter dendrite length and $R_{i}$ of the soma to $1 \Omega \mathrm{cm}$ (Wang et al., 2010). In accordance with previous models, we set the specific membrane capacitance $\left(C_{m}\right)$ of the dendrite to $1.3 \mu \mathrm{F} \mathrm{cm}{ }^{-2}$ to account for dendritic spines, which were not modeled explicitly, and to $1.7 \mu \mathrm{F} \mathrm{cm}^{-2}$ for the soma (Wang et al., 2010). The leak conductance (gleak) of the dendrite was set to $0.126 \mathrm{mS} / \mathrm{cm}^{2}$ (corresponding to a passive membrane resistivity $\left(R_{m}\right)$ of $8000 \mathrm{Ohm} \mathrm{cm}{ }^{2}$ ) (Branco et al., 2010; Branco and Häusser, 2011; Smith et al., 2013; Ujfalussy et al., 2018), gleak of the soma was set to 0.0379 $\mathrm{mS} / \mathrm{cm}^{2}$ (Lajeunesse et al., 2013), and $\mathrm{V}_{\mathrm{m}}$ was set to $-70 \mathrm{mV}$ in accordance with our electrophysiological recordings. To generate action potentials at the soma, we inserted NEURON's custom Hodgkin-andHuxley-spiking mechanism at the somatic compartment and used its default values for the active voltage-gated potassium $\left(\mathrm{g}^{\mathrm{k}}\right.$ of $\left.0.036 \mathrm{~S} / \mathrm{cm}^{2}\right)$ and sodium conductance $\left(\mathrm{g}^{\mathrm{Na}}\right.$ of $\left.0.12 \mathrm{~S} / \mathrm{cm}^{2}\right)$.

We inserted 270 synaptic conductances on the dendritic compartment, whose spike times, synaptic weights and short-term plasticity parameters were set as described in the following sections. Briefly, we first constructed 270 spike trains whose pairwise correlation coefficients and firing rates reproduced in vivo observations from rodent L2/3 (see Results). We then assigned these spike trains with EPSP amplitudes and corresponding paired-pulse ratios that reproduced our in vitro data.

\section{Generating input spike trains with temporal correlations following in vivo data}

We generated 270 input spike trains whose pairwise correlation coefficients matched the in vivo data reported by Cossell et al. (2014), i.e., the minority of (strong) input spike trains exhibited high pairwise correlation coefficients, while the remaining majority of (weak) input spike trains were subsequently less correlated. We first generated a template spike train of $10 \mathrm{~s}$ duration that exhibited a sparse and irregular temporal structure by using an inhomogeneous Poisson renewal process and sampling interspike interval durations from a gamma distribution (shape $\mathrm{k}=1.1$, inter-spike interval mean of $40 \mathrm{~ms}$ ) at $1 \mathrm{~ms}$ time steps, which resulted in an average firing rate of $25 \mathrm{~Hz}$. We convolved the template spike train with Gaussian envelopes of different standard deviations ( $\sigma$ Gaussian) to generate a set of 270 new 
spike trains with precisely defined correlation statistics (Destexhe and Paré, 1999; Hô and Destexhe, 2000; Azouz, 2005). We divided the 270 inputs into strong ( $n=35$, i.e., $13 \%$ of inputs) and weak inputs ( $n=235$, i.e., $87 \%$ of inputs) based on the relationship between EPSP amplitude and short-term plasticity we had found in vitro (i.e., synapses with EPSP amplitudes $>2 \mathrm{mV}$ (10 / 74 synaptic connections, i.e., $13.5 \%$ ) were exclusively depressing, while synapses with EPSP amplitudes $<2 \mathrm{mV}$ displayed the full range of short-term plasticity). In order to set up these two populations of input spike trains with corresponding temporal correlation statistics, we sampled oGaussian from two uniform distributions for strong ( $\sigma$ Gaussian between 5 and $10 \mathrm{~ms}, \mathrm{n}=35$ ) and weak synaptic inputs ( $\sigma$ Gaussian between 10 and $100 \mathrm{~ms}, \mathrm{n}=235$ ) (Azouz, 2005). The resulting 270 бGaussian values were ranked and assigned to the 270 input spike trains. For each one of the 270 input spike trains, we convolved the spike times of the template spike train with a Gaussian envelope whose standard deviation was set by each spike train's respective $\sigma$ Gaussian. By doing so, for each spike train, we obtained a $10 \mathrm{~s}$ time course consisting of a sum of Gaussian distributions representing the respective spike probability over time.

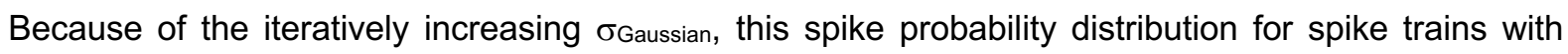
increasing indices continuously broadens and flattens with respect to the template spike train. We then generated the discrete spike times for each input spike train by drawing spike times from these timedependent spike probability distributions using an inhomogeneous Poisson process. The resulting 270 spike trains had continuously lower pairwise correlation coefficients with the template spike train.

Finally, we accounted for the fact that, in barrel cortex in vivo, correlated synaptic inputs tend to fire at higher frequencies, while uncorrelated inputs fire at lower rates (O'Connor et al., 2010; Cossell et al., 2015). We parametrized the lognormal firing rate distribution measured by O'Conner et al. (2010) in mouse barrel cortex L2/3 in vivo (mean \pm s.d.: $4.16 \pm 8.33 \mathrm{~Hz}$ ) and drew 270 random 'target firing rates' from it. These values were ranked and assigned to the 270 input spike trains, such that spike trains with higher pairwise correlations with the template spike train also displayed higher target firing rates. We then removed stochastically individual spikes from each input spike train such that the average firing rate of each spike train matched the respective target firing rate.

After the 270 input spike trains had been generated in this manner, we verified that their pairwise correlation coefficients (Cossell et al., 2015) and firing rates (O'Connor et al., 2010) matched experimental data obtained in rodent L2/3 in vivo (see Results, Fig. 4). This process was repeated 100 times to generate 100 different sets of spike trains to be run in the model.

\section{Generating EPSP amplitude and corresponding paired-pulse ratio distributions}

To assign realistic EPSP amplitudes to the 270 model inputs, we parametrized the EPSP amplitude distribution we measured in regular-spiking neurons in vitro with a lognormal distribution (Fig. $1 \mathrm{D}$, see Results) and randomly drew 270 EPSP amplitude values from it. We then generated corresponding paired-pulse ratios for these 270 EPSP amplitudes by parametrized the relationship between the second pulse $\left(\mathrm{EPSP}_{2}\right)$ and the first pulse (i.e., the EPSP amplitude) of the paired-pulse stimulation paradigm that we had recorded in vitro (Fig. $1 \mathrm{C}$ ) with an exponential decay function. Critically, the jitter of the experimentally recorded $\mathrm{EPSP}_{2}$ values around this fitted curve did not differ significantly from a Gaussian distribution (non-parametric Kolmogorov Smirnov Test, p value of 0.48 ) with a mean \pm s.d. of 
$1.6 * 10^{-9} \pm 0.192$. This standard deviation captures the natural variance of the ratio of EPSP 2 to EPSP 1 and was subsequently used to generate our modeling data. For each of the selected 270 EPSP amplitudes, we first assigned a corresponding $\mathrm{EPSP}_{2}$ by using the value predicted by the fitted exponential decay function for the given EPSP 1 (i.e., the EPSP amplitude). We then added variance to the selected value as a number drawn from a random Gaussian process with a mean of 0 and a standard deviation of 0.192 . Finally, we verified that the resulting EPSP distribution, paired-pulse ratio distribution, and their mapping corresponded to our in vitro recording data (see Results).

\section{Modeling short-term plasticity dynamically during presynaptic spike trains}

The paired-pulse ratio captures a synapse's short-term plasticity response for two subsequent release events at a stereotypical time interval. To model short-term plasticity dynamically for ongoing activation during spike trains with variable inter-spike intervals, we formalized the short-term plasticity properties of our synapses into a general form by utilizing the widely-used extended Tsodyks-Markram model (Markram et al., 1998; Tsodyks et al., 1998):

$$
\begin{aligned}
& \frac{d R(t)}{d t}=\frac{1-R(t)}{\tau_{\text {rec }}}-u(t) \cdot R(t) \cdot \delta\left(t-t_{\text {sp }}\right) \\
& \frac{d u(t)}{d t}=\frac{U-u(t)}{\tau_{\text {facil }}}+f(1-u(t)) \cdot \delta\left(t-t_{\text {sp }}\right)
\end{aligned}
$$

Briefly, short-term depression (equation 1 ) is modeled as the depletion of the synaptic vesicle pool available for release $R(t)$, with $u(t) \cdot R(t)$ following a preceding release event at time $t_{s p}$, which is counterbalanced by vesicle pool recovery at a time constant $\tau_{\text {rec }}$. Short-term facilitation (equation 2 ) is modeled as an increase in release probability $u(t)$, with $f(1-u(t))$ following a preceding spike at $t_{s p}$, which decays to the baseline release probability $U$ with a time constant $\tau_{\text {facil }}$. Thus, a continuum of synaptic depression to facilitation can be modeled by specifying the values of the parameter set $\Theta=$ $\left\{\tau_{\text {rec }}, \tau_{\text {facil }}, U, f\right\}$ (Costa et al., 2013; Ghanbari et al., 2017).

To do so, we derived $\Theta$ for each one of the 270 model synapses as a function of their pairedpulse ratio, as follows. A computationally optimized form of equations (1) and (2) was derived by Costa et al. (2013) by integrating between spikes $n$ and $n+1$ at time $\Delta t n_{n}$ apart:

$$
\begin{gathered}
R_{n+1}=1-\left(1-R_{n}\left(1-u_{n}\right)\right) e^{-\frac{\Delta t_{n}}{\tau_{\text {rec }}}} \\
u_{n+1}=U+\left(u_{n}+f\left(1-u_{n}\right)-U\right) e^{-\frac{\Delta t_{n}}{\tau_{\text {facil }}}}
\end{gathered}
$$

The EPSP amplitude at spike $n$ can be calculated as:

$$
\operatorname{EPSP}_{n}=A \cdot R_{n} u_{n}
$$


(Markram et al., 1998), where $A$ is an adjustable weight parameter that convolves phenomenologically several physiological strength parameters, such as the number of release sites, quantal size, and cable filtering properties. The paired pulse ratio PPR is the ratio of the EPSP at spike $n+1$ and the EPSP at spike $n$ :

$$
P P R=\frac{A \cdot R_{n+1} u_{n+1}}{A \cdot R_{n} u_{n}}
$$

At time $t=0$, when no preceding spike occurred, the steady-state value of $R_{n}=1$ and of $u_{n}=U$, and equation (6) can be simplified to:

$$
P P R_{0}=\frac{R_{n+1} u_{n+1}}{U}
$$

By inserting equations ( 3 ) and (4) for $R_{n+1}$ and $u_{n+1}$, we can rewrite equation (7) as

$$
P P R_{0}=\frac{\left(1-U e^{-\frac{\Delta t_{n}}{\tau_{r e c}}}\right)\left(U+f(1-U) e^{-\frac{\Delta t_{n}}{\tau_{f a c i l}}}\right)}{U}
$$

Critically, $P P R_{0}$ in equation (8) at $\Delta t n_{n}=20 \mathrm{~ms}$ (i.e., $P P R_{0}^{20 m s}$ ) describes exactly our experimental paired-pulse stimulation protocol. This allowed us to obtain a parameter set $\Theta$ for each synapse as a function of its $20 \mathrm{~ms}$ paired-pulse ratio.

\section{Defining the short-term plasticity parameter set $\Theta$ for each synapse}

To do so, we varied $\Theta$ on a continuum ranging from strong depression to strong facilitation according to Costa et al. (2013) (Table 1), which resulted in a large dataset of uniquely defined $\Theta s$ and corresponding $P P R_{0}^{20 m s}$ values. For each of our 270 model synapses, we then chose the parameter set $\Theta$, whose resulting $P P R_{0}^{20 m s}$ value matched most closely the paired-pulse ratio we had previously assigned to that synapse (see above). By obtaining a unique parameter set $\Theta$ for each synapse, we could then compute its $R_{n+1}$ and $u_{n+1}$ during continuous spike trains using equations (3) and (4), respectively.

Table 1. Parameter sets $\Theta$ for strongly depressing and strongly facilitation synapses, adopted from Costa et al. (2013).

\begin{tabular}{lccccc}
\hline Synaptic short-term plasticity & $\tau_{\text {rec }}$ & $\tau_{\text {facil }}$ & $\mathrm{U}$ & $\mathrm{f}$ & $P P R_{0}^{20 \mathrm{~ms}}$ \\
\hline Strong depression & $1700 \mathrm{~ms}$ & $20 \mathrm{~ms}$ & 0.7 & 0.05 & 0.3 \\
Strong facilitation & $20 \mathrm{~ms}$ & $1700 \mathrm{~ms}$ & 0.1 & 0.11 & 1.8 \\
\hline
\end{tabular}

\section{Modeling EPSP amplitude and paired-pulse ratio in the NEURON simulation}

We modeled the input synapses by using the ExpSyn point process in NEURON, which allows for the synaptic strength to be set precisely by means of a weight parameter. We defined the weight parameter 
as the product of the desired somatic EPSP amplitude and a scaling factor. To determine this scaling factor, we generated a single test spike for each of the 270 EPSP amplitudes (using its respective desired EPSP amplitude) and measured the resulting somatic EPSP amplitude. We found that the ratio of the desired EPSP / test EPSP was a constant factor across all 270 input synapses, which allowed us to use this ratio as the universal scaling factor.

By deriving paired-pulse ratios using equation (6), we were able to adjust the EPSP amplitudes dynamically in the simulation to incorporate short-term plasticity. To cross-check again that the simulated EPSP amplitudes and short-term plasticity properties reproduced the desired values, each synapse in the NEURON simulation was activated with two pulses at a $20 \mathrm{~ms}$ inter-spike interval and the somatic EPSP amplitudes and paired-pulse ratios measured at the soma of the model neuron. Reassuringly, we found that the resulting somatic EPSP amplitude distribution and paired-pulse ratio distribution exactly matched the target distributions we had generated (as descried above).

\section{Modeling the interplay of synaptic strength, short-term plasticity, and temporal correlation in presynaptic spike trains}

After the model was set up in this manner, we simulated the somatic voltage response of the model neuron following activation of the 270 input synapses with the corresponding presynaptic spike trains. We convolved the discrete spike times of the output spike train of the model neuron and each one of the 270 input spike trains into continuous functions with an exponential filter $(\tau=10 \mathrm{~ms}$ ) (van Rossum, 2001) and computed the pairwise Pearson's correlation coefficients between each input spike train and the output spike train. Additionally, we quantified the input-output relationship of the model neuron as the probability of spike generation as a function of the number of coincident inputs in the $20 \mathrm{~ms}$ time window preceding the output spike. As described in the Results, we then manipulated the respective population of active synapses and their synaptic parameters in the simulation to investigate the interplay of synaptic strength, short-term plasticity, and temporal correlation in presynaptic spike trains. We computed mean correlation coefficients, input-output curves and corresponding $95 \%$ confidence intervals by repeating each simulation setup for the 100 sets of spike trains (see above). 


\section{Acknowledgements}

We would like to thank Kevan A.C. Martin for his inspiration, support, comments on the manuscript, and funding. We would like to thank Qendrasa Parduzzi for help with developing the NEURON model. As members of the Institute of Neuroinformatics, the authors are signatories of the Basel Declaration. This work was supported by funding from the University of Zurich to Kevan A.C. Martin and by funding of the Swiss National Science Foundation to Gregor Schuhknecht.

\section{Author Contributions}

B.E. and G.F.P.S. designed research,

M.O.B. performed electrophysiology experiments and histology,

M.O.B. and G.F.P.S. analyzed electrophysiology data,

A.G. and B.E. developed the NEURON model,

A.G. analyzed modeling data,

B.E. and G.F.P.S. supervised the work,

G.F.P.S. wrote the paper with input from all authors. 


\section{References}

Abbott LF, Regehr WG (2004) Synaptic computation. Nature 431:796-803.

Abbott LF, Varela JA, Sen K, Nelson SB (1997) Synaptic depression and cortical gain control. Science 275:221-224.

Azouz R (2005) Dynamic spatiotemporal synaptic integration in cortical neurons: neuronal gain, revisited. J Neurophysiol 94:2785-2796.

Banitt Y, Martin KAC, Segev I (2007) A biologically realistic model of contrast invariant orientation tuning by thalamocortical synaptic depression. J Neurosci 27:10230-10239.

Bosking WH, Zhang Y, Schofield B, Fitzpatrick D (1997) Orientation selectivity and the arrangement of horizontal connections in tree shrew striate cortex. J Neurosci 17:2112-2127.

Boudreau CE, Ferster D (2005) Short-term depression in thalamocortical synapses of cat primary visual cortex. J Neurosci 25:7179-7190.

Branco T, Clark BA, Häusser M (2010) Dendritic discrimination of temporal input sequences in cortical neurons. Science 329:1671-1675.

Branco T, Häusser M (2011) Synaptic integration gradients in single cortical pyramidal cell dendrites. Neuron 69:885-892.

Bruno RM, Sakmann B (2006) Cortex is driven by weak but synchronously active thalamocortical synapses. Science 312:1622-1627.

Bulsara A, Jacobs EW, Zhou T, Moss F, Kiss L (1991) Stochastic resonance in a single neuron model: Theory and analog simulation. J Theor Biol 152:531-555.

Buzsáki G, Mizuseki K (2014) The log-dynamic brain: how skewed distributions affect network operations. Nat Rev Neurosci 15:264-278.

Castro-Alamancos MA, Oldford E (2002) Cortical sensory suppression during arousal is due to the activity-dependent depression of thalamocortical synapses. J Physiol 541:319-331.

Chance FS, Abbott LF, Reyes AD (2002) Gain modulation from background synaptic input. Neuron 35:773-782.

Chance FS, Nelson SB, Abbott LF (1998) Synaptic depression and the temporal response characteristics of V1 cells. J Neurosci 18:4785-4799.

Chapeau-Blondeau F, Rousseau D (2002) Noise improvements in stochastic resonance: from signal amplification to optimal detection. Fluct Noise Lett 2:L221-L233.

Chung S, Li X, Nelson SB (2002) Short-term depression at thalamocortical synapses contributes to rapid adaptation of cortical sensory responses in vivo. Neuron 34:437-446.

Cossell L, lacaruso MF, Muir DR, Houlton R, Sader EN, Ko H, Hofer SB, Mrsic-Flogel TD (2015) Functional organization of excitatory synaptic strength in primary visual cortex. Nature 518:399-403.

Costa RP, Sjostrom PJ, van Rossum MCW (2013) Probabilistic inference of short-term synaptic plasticity in neocortical microcircuits. Front Comput Neurosci 7:75.

Crochet S, Poulet JFA, Kremer Y, Petersen CCH (2011) Synaptic mechanisms underlying sparse coding of active touch. Neuron 69:1160-1175.

de Kock CPJ, Bruno RM, Spors H, Sakmann B (2007) Layer- and cell-type-specific suprathreshold stimulus representation in rat primary somatosensory cortex: Sensory responses throughout barrel cortex. J Physiol 581:139-154.

de Kock CPJ, Sakmann B (2009) Spiking in primary somatosensory cortex during natural whisking in awake head-restrained rats is cell-type specific. PNAS 106:16446-16450.

Destexhe A, Paré D (1999) Impact of network activity on the integrative properties of neocortical pyramidal neurons in vivo. J Neurophysiol 81:1531-1547. 
Díaz-Quesada M, Martini FJ, Ferrati G, Bureau I, Maravall M (2014) Diverse thalamocortical short-term plasticity elicited by ongoing stimulation. J Neurosci 34:515-526.

Durand DM, Kawaguchi M, Mino H (2013) Reverse stochastic resonance in a hippocampal CA1 neuron model. In: 35th Annual International Conference of the IEEE Engineering in Medicine and Biology Society (EMBC), pp 5242-5245.

Faisal AA, Selen LPJ, Wolpert DM (2008) Noise in the nervous system. Nat Rev Neurosci 9:292-303.

Feldmeyer D, Lübke J, Sakmann B (2006) Efficacy and connectivity of intracolumnar pairs of layer 2/3 pyramidal cells in the barrel cortex of juvenile rats. J Physiol 575:583-602.

Ferrarese L, Jouhanneau J-S, Remme MWH, Kremkow J, Katona G, Rózsa B, Schreiber S, Poulet JFA (2018) Dendrite-specific amplification of weak synaptic input during network activity in vivo. Cell Rep 24:3455-3465.e5.

Fortune ES, Rose GJ (2000) Short-term synaptic plasticity contributes to the temporal filtering of electrosensory information. J Neurosci 20:7122-7130.

Fortune ES, Rose GJ (2001) Short-term synaptic plasticity as a temporal filter. Trends Neurosci 24:381385.

Ghanbari A, Malyshev A, Volgushev M, Stevenson IH (2017) Estimating short-term synaptic plasticity from pre- and postsynaptic spiking. PLOS Comput Biol 13:e1005738.

Gilbert CD, Wiesel TN (1989) Columnar specificity of intrinsic horizontal and corticocortical connections in cat visual cortex. J Neurosci 9:2432-2442.

Girman SV, Sauvé Y, Lund RD (1999) Receptive field properties of single neurons in rat primary visual cortex. J Neurophysiol 82:301-311.

Goetz L, Roth A, Häusser M (2021) Active dendrites enable strong but sparse inputs to determine orientation selectivity. PNAS 118(30).

Guzman SJ, Schlögl A, Schmidt-Hieber C (2014) Stimfit: quantifying electrophysiological data with Python. Front Neuroinform 8:16.

Hines ML, Carnevale NT (1997) The NEURON simulation environment. Neural Comput 9:1179-1209.

Hô N, Destexhe A (2000) Synaptic background activity enhances the responsiveness of neocortical pyramidal neurons. J Neurophysiol 84:1488-1496.

Holler S, Köstinger G, Martin KAC, Schuhknecht GFP, Stratford KJ (2021) Structure and function of a neocortical synapse. Nature 591:111-116.

Hubel DH, Wiesel TN (1962) Receptive fields, binocular interaction and functional architecture in the cat's visual cortex. J Physiol 160:106-154.

Jouhanneau J-S, Kremkow J, Dorrn AL, Poulet JFA (2015) In vivo monosynaptic excitatory transmission between layer 2 cortical pyramidal neurons. Cell Rep 13:2098-2106.

Karimi A, Odenthal J, Drawitsch F, Boergens KM, Helmstaedter M (2020) Cell-type specific innervation of cortical pyramidal cells at their apical dendrites. eLife 9:e46876.

Kerr JND, de Kock CPJ, Greenberg DS, Bruno RM, Sakmann B, Helmchen F (2007) Spatial organization of neuronal population responses in layer $2 / 3$ of rat barrel cortex. J Neurosci 27:13316-13328.

Koulakov AA, Hromádka T, Zador AM (2009) Correlated connectivity and the distribution of firing rates in the neocortex. J Neurosci 29:3685-3694.

Lajeunesse F, Kröger H, Timofeev I (2013) Regulation of AMPA and NMDA receptor-mediated EPSPs in dendritic trees of thalamocortical cells. J Neurophysiol 109:13-30.

Larkum ME (2004) Top-down dendritic input increases the gain of layer 5 pyramidal neurons. Cereb Cortex 14:1059-1070. 
Lefort S, Petersen CCH (2017) Layer-dependent short-term synaptic plasticity between excitatory neurons in the $\mathrm{C} 2$ barrel column of mouse primary somatosensory cortex. Cereb Cortex 27:3869-3878.

Lefort S, Tomm C, Floyd Sarria J-C, Petersen CCH (2009) The excitatory neuronal network of the C2 barrel column in mouse primary somatosensory cortex. Neuron 61:301-316.

Lisman J (1997) Bursts as a unit of neural information: making unreliable synapses reliable. Trends Neurosci 20:38-43.

London M, Schreibman A, Häusser M, Larkum ME, Segev I (2002) The information efficacy of a synapse. Nat Neurosci 5:332-340.

Mainen ZF, Sejnowski TJ (1996) Influence of dendritic structure on firing pattern in model neocortical neurons. Nature 382:363-366.

Malach R, Tootell RBH, Malonek D (1994) Relationship between orientation domains, cytochrome oxidase stripes, and intrinsic horizontal connections in squirrel monkey area V2. Cereb Cortex 4:151-165.

Markram H, Lübke J, Frotscher M, Roth A, Sakmann B (1997) Physiology and anatomy of synaptic connections between thick tufted pyramidal neurones in the developing rat neocortex. J Physiol 500:409-440.

Markram H, Wang Y, Tsodyks M (1998) Differential signaling via the same axon of neocortical pyramidal neurons. PNAS 95:5323-5328.

Martin KAC, Roth S, Rusch ES (2014) Superficial layer pyramidal cells communicate heterogeneously between multiple functional domains of cat primary visual cortex. Nat Commun 5, 5252.

Martin KAC, Schröder S (2016) Phase locking of multiple single neurons to the local field potential in cat V1. J Neurosci 36:2494-2502.

McDonnell MD, Abbott D (2009) What Is stochastic resonance? Definitions, misconceptions, debates, and its relevance to biology. PLOS Comput Biol 5:e1000348.

Niell CM, Stryker MP (2010) Modulation of visual responses by behavioral state in mouse visual cortex. Neuron 65:472-479.

O'Connor DH, Peron SP, Huber D, Svoboda K (2010) Neural activity in barrel cortex underlying vibrissabased object localization in mice. Neuron 67:1048-1061.

Ohki K, Chung S, Ch'ng YH, Kara P, Reid RC (2005) Functional imaging with cellular resolution reveals precise micro-architecture in visual cortex. Nature 433:597-603.

Petersen $\mathrm{CCH}$, Crochet $\mathrm{S}$ (2013) Synaptic computation and sensory processing in neocortical layer 2/3. Neuron 78:28-48.

Pinsky PE, Rinsky J (1994) Intrinsic and network rhythmogenesis in a reduced Traub model for CA3 neurons. J Comput Neurosci 1:39-60.

Reyes A, Sakmann B (1999) Developmental switch in the short-term modification of unitary EPSPs evoked in layer 2/3 and layer 5 pyramidal neurons of rat neocortex. J Neurosci 19:3827-3835.

Rothman JS, Cathala L, Steuber V, Silver RA (2009) Synaptic depression enables neuronal gain control. Nature 457:1015-1018.

Sakata S, Harris KD (2009) Laminar structure of spontaneous and sensory-evoked population activity in auditory cortex. Neuron 64:404-418.

Salinas E, Sejnowski TJ (2001) Correlated neuronal activity and the flow of neural information. Nat Rev Neurosci 2:539-550.

Sarid L, Feldmeyer D, Gidon A, Sakmann B, Segev I (2013) Contribution of intracolumnar layer 2/3-tolayer $2 / 3$ excitatory connections in shaping the response to whisker deflection in rat barrel cortex. Cereb Cortex 25:849-858. 
Sato TR, Gray NW, Mainen ZF, Svoboda K (2007) The functional microarchitecture of the mouse barrel cortex. PLOS Biol 5:e189.

Scholl B, Thomas CI, Ryan MA, Kamasawa N, Fitzpatrick D (2020) Cortical response selectivity derives from strength in numbers of synapses. Nature 590:111-114.

Schoonover CE, Tapia J-C, Schilling VC, Wimmer V, Blazeski R, Zhang W, Mason CA, Bruno RM (2014) Comparative strength and dendritic organization of thalamocortical and corticocortical synapses onto excitatory layer 4 neurons. J Neurosci 34:6746-6758.

Schüz A, Palm G (1989) Density of neurons and synapses in the cerebral cortex of the mouse. J Comp Neurol 286:442-455.

Seeman SC et al. (2018) Sparse recurrent excitatory connectivity in the microcircuit of the adult mouse and human cortex. eLife 7:e37349.

Silver RA (2010) Neuronal arithmetic. Nat Rev Neurosci 11:474-489.

Sincich LC, Blasdel GG (2001) Oriented axon projections in primary visual cortex of the monkey. J Neurosci 21:4416-4426.

Smith SL, Smith IT, Branco T, Häusser M (2013) Dendritic spikes enhance stimulus selectivity in cortical neurons in vivo. Nature 503:115-120.

Song S, Sjöström PJ, Reigl M, Nelson S, Chklovskii DB (2005) Highly nonrandom features of synaptic connectivity in local cortical circuits. PLOS Biol 3:e68.

Stratford KJ, Tarczy-Hornoch K, Martin KAC, Bannister NJ, Jack JJB (1996) Excitatory synaptic inputs to spiny stellate cells in cat visual cortex. Nature 382:258-261.

Stuart G, Spruston N (1998) Determinants of voltage attenuation in neocortical pyramidal neuron dendrites. J Neurosci 18:3501-3510.

Tarczy-Hornoch K, Martin KAC, Stratford KJ, Jack JJB (1999) Intracortical excitation of spiny neurons in layer 4 of cat striate cortex in vitro. Cereb Cortex 9:833-843.

Tsodyks M, Pawelzik K, Markram H (1998) Neural networks with dynamic synapses. Neural Comput 10:821-835.

Ujfalussy BB, Makara JK, Lengyel M, Branco T (2018) Global and multiplexed dendritic computations under in vivo-like conditions. Neuron 100:579-592.

van Rossum MCW (2001) A novel spike distance. Neural Comput 13:751-763.

Wang H-P, Spencer D, Fellous J-M, Sejnowski TJ (2010) Synchrony of thalamocortical inputs maximizes cortical reliability. Science 328:106-109.

Waters J, Helmchen F (2006) Background synaptic activity Is sparse in neocortex. J Neurosci 26:82678277.

Yi G, Wang J, Wei X, Deng B (2017) Action potential initiation in a two-compartment model of pyramidal neuron mediated by dendritic Ca2+ spike. Sci Rep 7:45684.

Znamenskiy P, Kim M-H, Muir DR, lacaruso MF, Hofer SB, Mrsic-Flogel TD (2018) Functional selectivity and specific connectivity of inhibitory neurons in primary visual cortex. bioRxiv:294835. 